\title{
ON OBJECTIVE AND STRONG OBJECTIVE CONSISTENT ESTIMATES OF UNKNOWN PARAMETERS FOR STATISTICAL STRUCTURES IN A POLISH GROUP ADMITTING AN INVARIANT METRIC
}

\section{MURMAN KINTSURASHVILI, TENGIZ KIRIA and GOGI PANTSULAIA}

Department of Mathematics

Georgian Technical University

Kostava Street. 77

Tbilisi DC 0175

Republic of Georgia

e-mail: m.kintsurashvili@gtu.ge

t.kiria@gtu.ge

g.pantsulaia@gtu.ge

\begin{abstract}
By using the notion of a Haar ambivalent set introduced by Balka et al. [1], essentially new classes of statistical structures having objective and strong objective estimates of unknown parameters are introduced in a Polish nonlocally-compact group admitting an invariant metric and relations between them are studied in this paper. An example of such a weakly separated statistical structure is constructed for which a question asking "whether there exists a consistent estimate of an unknown parameter" is not solvable within the theory $(Z F) \&(D C)$. A question asking "whether there exists an objective consistent estimate of an unknown parameter for any statistical structure in a non-locally compact Polish group with an invariant metric when subjective one
\end{abstract}

2010 Mathematics Subject Classification: Primary 62-02; Secondary 62D05.

Keywords and phrases: an objective infinite-sample consistent estimate, shy set, Haar ambivalent set, Polish group.

Received March 19, 2015

(C) 2015 Scientific Advances Publishers 
exists" is answered positively when there exists at least one such a parameter the pre-image of which under this subjective estimate is a prevalent. These results extend recent results of authors. Some examples of objective and strong objective consistent estimates in a compact Polish group $\{0 ; 1\}^{N}$ are considered in this paper.

\section{Introduction}

In order to explain a big gap between the theory of mathematical statistics and results of hypothesis testing, concepts of subjective and objective infinite sample consistent estimates of a useful signal in the linear one-dimensional stochastic model were introduced in [18]. This approach essentially uses the concept of Haar null sets in Polish topological vector spaces introduced by Christensen [2].

The Polish topological vector space $\mathbf{R}^{N}$ of all real-valued sequences (equivalently, of infinite samples) equipped with Tychonoff metric plays a central role in the theory of statistical decisions because a definition of any consistent estimate of an unknown parameter in various stochastic models without infinite samples is simply impossible.

Let explain from the point of view of the theory of Haar null sets in $\mathbf{R}^{N}$ some confusions which were described by Nunnally [14] and Cohen [4]:

Let $x_{1}, x_{2}, \cdots$ be an infinite sample obtained by observation on independent and normally distributed real-valued random variables with parameters $(\theta, 1)$, where $\theta$ is an unknown mean and the variance is equal to 1 . Using this infinite sample, we want to estimate an unknown mean. If we denote by $\mu_{\theta}$ a linear Gaussian measure on $\mathbf{R}$ with the probability density $\frac{1}{\sqrt{2 \pi}} e^{-\frac{(x-\theta)^{2}}{2}}$, then the triplet

$$
\left(\mathbf{R}^{N}, \mathcal{B}\left(\mathbf{R}^{N}\right), \mu_{\theta}^{N}\right)_{\theta \in R}
$$


stands a statistical structure described our experiment, where $\mathcal{B}\left(\mathbf{R}^{N}\right)$ denotes the $\sigma$-algebra of Borel subsets of $\mathbf{R}^{N}$. By virtue of the strong law of large numbers, we know that the condition

$$
\mu_{\theta}^{N}\left(\left\{\left(x_{k}\right)_{k \in N}:\left(x_{k}\right)_{k \in N} \in \mathbf{R}^{N} \& \lim _{n \rightarrow \infty} \frac{\sum_{k=1}^{n} x_{k}}{n}=\theta\right\}\right)=1
$$

holds true for each $\theta \in \mathbf{R}$.

Take into account the validity of (1.2), for construction of a consistent infinite sample estimation of an unknown parameter $\theta$ a mapping $T$ defined by

$$
T\left(\left(x_{k}\right)_{k \in N}\right)=\lim _{n \rightarrow \infty} \frac{\sum_{k=1}^{n} x_{k}}{n}
$$

is used by statisticians. As usual, null hypothesis significance testing in the case $H_{0}: \theta=\theta_{0}$ assumes the following procedure: If an infinite sample $\left(x_{k}\right)_{k \in N} \in T^{-1}\left(\theta_{0}\right)$, then $H_{0}$ hypothesis is accepted and $H_{0}$ hypothesis is rejected, otherwise. There naturally arises a question asking whether can be explained Cohen statement [4]: “... Dont look for a magic alternative to NHST [null hypothesis significance testing] ... It does not exist." Notice that a set $S$ of all infinite samples $\left(x_{k}\right)_{k \in N}$ for which there exist finite limits of arithmetic means of their first $n$ elements constitutes a proper Borel measurable vector subspace of $\mathbf{R}^{N}$. Following Christensen [2], each proper Borel measurable vector subspace of an arbitrary Polish topological vector space is Haar null set and since $S$ is a Borel measurable proper vector subspace of $\mathbf{R}^{N}$ we claim that the mapping $T$ is not defined for "almost every" (in the sense of Christensen ${ }^{1}$

${ }^{1}$ We say that a sentence $P(\cdot)$ formulated in term of an element of a Polish group $G$ is true for "almost every" element of $G$ if a set of all elements $g \in G$ for which $P(g)$ is false constitutes a Haar null set in $G$. 
[2]) infinite sample. The latter relation means that for "almost every" infinite sample we reject null hypothesis $H_{0}$. This discussion can be used also to explain Nunnally's [14] following conjecture: "If the decisions are based on convention they are termed arbitrary or mindless while those not so based may be termed subjective. To minimize type II errors, large samples are recommended. In psychology, practically all null hypotheses are claimed to be false for sufficiently large samples so ... it is usually nonsensical to perform an experiment with the sole aim of rejecting the null hypothesis".

Now, let $T_{1}: \mathbf{R}^{N} \rightarrow R$ be another infinite sample consistent estimate of an unknown parameter $\theta$ in the above mentioned model, i.e.,

$$
\mu_{\theta}^{N}\left(\left\{\left(x_{k}\right)_{k \in N}:\left(x_{k}\right)_{k \in N} \in \mathbf{R}^{N} \& T_{1}\left(\left(x_{k}\right)_{k \in N}\right)=\theta\right\}\right)=1,
$$

for each $\theta \in \mathbf{R}$. Here naturally arises a question asking what are those additional conditions imposed on the estimate $T_{1}$ under which the abovedescribed confusions will be settled.

In this direction, first, notice that there must be no a parameter $\theta_{0} \in R$ for which $T_{1}^{-1}\left(\theta_{0}\right)$ is Haar null set, because then for "almost every" infinite sample null hypothesis $H_{0}: \theta=\theta_{0}$ will be rejected. Second, there must be no a parameter $\theta_{1} \in \mathbf{R}$ for which $T_{1}^{-1}\left(\theta_{1}\right)$ is a prevalent set (equivalently, a complement of a Haar null set) because then for "almost every" infinite sample null hypothesis $H_{0}: \theta=\theta_{2}$ will be rejected for each $\theta_{2} \neq \theta_{1}$. This observations lead us to additional conditions imposed on the estimate $T_{1}$, which assumes that $T_{1}^{-1}(\theta)$ must be neither Haar null nor prevalent for each $\theta \in \mathbf{R}$. Following [1], a set which is neither Haar null nor prevalent is called a Haar ambivalent set. Such estimates firstly were adopted as objective infinite sample consistent estimates of a useful signal in the linear one-dimensional stochastic model (see [27], Theorem 4.1, p. 482). 
It was proved in [27] that $T_{n}: \mathbf{R}^{n} \rightarrow \mathbf{R}(n \in N)$ defined by

$$
T_{n}\left(x_{1}, \cdots, x_{n}\right)=-F^{-1}\left(n^{-1} \#\left(\left\{x_{1}, \cdots, x_{n}\right\} \cap(-\infty ; 0]\right)\right),
$$

for $\left(x_{1}, \cdots, x_{n}\right) \in \mathbf{R}^{n}$, is a consistent estimator of a useful signal $\theta$ in one-dimensional linear stochastic model

$$
\xi_{k}=\theta+\Delta_{k}(k \in N),
$$

where \# (.) denotes a counting measure, $\Delta_{k}$ is a sequence of independent identically distributed random variables on $\mathbf{R}$ with strictly increasing continuous distribution function $F$ and expectation of $\Delta_{1}$ does not exist. In this direction, the following two examples of simulations of linear onedimensional stochastic models have been considered.

Example 1.1 ([27], Example 4.1, p. 484). Since a sequence of real numbers $(\pi \times n-[\pi \times n])_{n \in N}$, where [.] denotes an integer part of a real number, is uniformly distributed on $(0,1)$ (see [10], Example 2.1, p.17), we claim that a simulation of a $\mu_{(\theta, 1)}$-equidistributed sequence $\left(x_{n}\right)_{n \leq M}$ on $R$ ( $M$ is a "sufficiently large" natural number and depends on a representation quality of the irrational number $\pi$ ), where $\mu_{(\theta, 1)}$ denotes a $\theta$-shift of the measure $\mu$ defined by distribution function $F$, can be obtained by the formula

$$
x_{n}=F_{\theta}^{-1}(\pi \times n-[\pi \times n]),
$$

for $n \leq M$ and $\theta \in R$, where $F_{\theta}$ denotes a distribution function corresponding to the measure $\mu_{\theta}$.

In this model, $\theta$ stands a "useful signal".

We set:

(i) $n$ - the number of trials;

(ii) $T_{n}$ - an estimator defined by the formula (1.5);

(iii) $\bar{X}_{n}$ - a sample average. 
Table 1. Estimates of the useful signal $\theta=1$ when the white noise is standard Gaussian random variable

\begin{tabular}{cccccc}
\hline$n$ & $T_{n}$ & $\bar{X}_{n}$ & $\mathrm{n}$ & $T_{n}$ & $\bar{X}_{n}$ \\
\hline 50 & 0.994457883 & 1.146952654 & 550 & 1.04034032 & 1.034899747 \\
100 & 1.036433389 & 1.010190601 & 600 & 1.036433389 & 1.043940988 \\
150 & 1.022241387 & 1.064790041 & 650 & 1.03313984 & 1.036321771 \\
200 & 1.036433389 & 1.037987511 & 700 & 1.030325691 & 1.037905202 \\
250 & 1.027893346 & 1.045296447 & 750 & 1.033578332 & 1.03728633 \\
300 & 1.036433389 & 1.044049728 & 800 & 1.03108705 & 1.032630945 \\
350 & 1.030325691 & 1.034339407 & 850 & 1.033913784 & 1.037321098 \\
400 & 1.036433389 & 1.045181911 & 900 & 1.031679632 & 1.026202323 \\
450 & 1.031679632 & 1.023083495 & 950 & 1.034178696 & 1.036669278 \\
500 & 1.036433389 & 1.044635371 & 1000 & 1.036433389 & 1.031131694 \\
\hline
\end{tabular}

When $F(x)$ is a standard Gaussian distribution function, by using Microsoft Excel we have obtained numerical data placed in Table 1. Notice that results of computations presented in Table 1 show us that both statistics $T_{n}$ and $\bar{X}_{n}$ give us a good estimates of the "useful signal" $\theta$ whenever a generalized "white noise" in that case has a finite absolute moment of the first order and its moment of the first order is equal to zero.

Now let $F$ be a linear Cauchy distribution function on $R$, i.e.,

$$
F(x)=\int_{-\infty}^{x} \frac{1}{\pi\left(1+t^{2}\right)} d t(x \in R) .
$$

Numerical data placed in Table 2 were obtaining by using Microsoft Excel and Cauchy distribution calculator of the high accuracy [8]. On the one hand, the results of computations placed in Table 2 do not contradict to the above mentioned fact that $T_{n}$ is a consistent estimator of the parameter $\theta=1$. On the other hand, we know that a sample average $\bar{X}_{n}$ does not work in that case because the mean and variance of the "white 
noise" (i.e., Cauchy random variable) are not defined. By this reason, attempts to estimate the "useful signal" $\theta=1$ by using the sample average will not be successful.

Table 2. Estimates of the useful signal $\theta=1$ when the white noise is Cauchy random variable

\begin{tabular}{cccccc}
\hline$n$ & $T_{n}$ & $\bar{X}_{n}$ & $n$ & $T_{n}$ & $\bar{X}_{n}$ \\
\hline 50 & 1.20879235 & 2.555449288 & 550 & 1.017284476 & 41.08688757 \\
100 & 0.939062506 & 1.331789564 & 600 & 1.042790358 & 41.30221291 \\
150 & 1.06489184 & 71.87525566 & 650 & 1.014605804 & 38.1800532 \\
200 & 1.00000000 & 54.09578271 & 700 & 1.027297114 & 38.03399768 \\
250 & 1.06489184 & 64.59240343 & 750 & 1.012645994 & 35.57956117 \\
300 & 1.021166379 & 54.03265563 & 800 & 1.015832638 & 35.25149408 \\
350 & 1.027297114 & 56.39846672 & 850 & 1.018652839 & 33.28723503 \\
400 & 1.031919949 & 49.58316089 & 900 & 1.0070058 & 31.4036155 \\
450 & 1.0070058 & 44.00842613 & 950 & 1.023420701 & 31.27321466 \\
500 & 1.038428014 & 45.14322051 & 1000 & 1.012645994 & 29.73405416 \\
\hline
\end{tabular}

In [27] has been established that the estimators $\widetilde{\lim } \widetilde{T_{n}}:=\inf _{n} \sup _{m \geq n}$ $\widetilde{T_{m}}$ and $\underline{\lim } \widetilde{T_{n}}:=\sup _{n} \inf _{m \geq n} \widetilde{T_{m}}$ are consistent infinite sample estimates of a useful signal $\theta$ in the model (1.6) (see [27], Theorem 4.2, p. 483). When we begin to study properties of these infinite sample estimators from the point of view of the theory of Haar null sets in $\mathbf{R}^{N}$, we observed a surprising and an unexpected fact for us that these both estimates are objective (see [20], Theorem 3.1).

As the described approach naturally divides a class of consistent infinite sample estimates into objective and subjective estimates should not seem excessively highly told our suggestion that each consistent infinite sample estimate must pass the theoretical test on the objectivity.

The present manuscript introduces the concepts of the theory of objective infinite sample consistent estimates in $\mathbf{R}^{N}$ and gives its extension to all non-locally-compact Polish groups admitting an invariant metric. 
The rest of this note is the following:

In Section 2, we give some notions and facts from the theory of Haar null sets in complete metric linear spaces and equidistributed sequences on the real axis $\mathbf{R}$. Concepts of objective and strong objective infinite sample consistent estimates for statistical structures are introduced also in this section. Section 3 presents a certain construction of the objective infinite sample consistent estimate of an unknown distribution function which generalises the recent results obtained in [27]. There is proved an existence of the infinite sample consistent estimate of an unknown distribution function $F(F \in \mathcal{F})$ for the family of Borel probability measures $\left\{p_{F}^{N}: F \in \mathcal{F}\right\}$, where $\mathcal{F}$ denotes the family of all strictly increasing and continuous distribution functions on $\mathbf{R}$ and $p_{F}^{N}$ denotes an infinite power of the Borel probability measure $p_{F}$ on $\mathbf{R}$ defined by $F$. Section 4 presents an effective construction of the strong objective infinite sample consistent estimate of the "useful signal" in a certain linear one-dimensional stochastic model. An infinite sample consistent estimate of an unknown probability density is constructed for the separated class of positive continuous probability densities and a problem about existence of an objective one is stated in Section 5. In Section 6, by using the notion of a Haar ambivalent set introduced in [1], essentially new classes of statistical structures having objective and strong objective estimates of an unknown parameter are introduced in a Polish nonlocally-compact group admitting an invariant metric and relations between them are studied in this section. An example of such a weakly separated statistical structure is constructed for which a question asking whether there exists a consistent estimate of an unknown parameter is not solvable within the theory $(Z F) \&(D C)$. These results extend recent results obtained in [19]. In addition, we extend the concept of objective and subjective consistent estimates introduced for $\mathbf{R}^{N}$ to all Polish groups and consider a question asking whether there exists an objective consistent estimate of an unknown parameter for any statistical 
structure in a non-locally compact Polish group with an invariant metric when subjective one exists. We show that this question is answered positively when there exists at least one such a parameter the pre-image of which under this subjective estimate is a prevalent. In Section 7, we consider some examples of objective and strong objective consistent estimates in a compact Polish group $\{0 ; 1\}^{N}$.

\section{Auxiliary Notions and Facts from Functional Analysis and Measure Theory}

Let $V$ be a complete metric linear space, by which we mean a vector space (real or complex) with a complete metric for which the operations of addition and scalar multiplication are continuous. When we speak of a measure on $V$ we will always mean a nonnegative measure that is defined on the Borel sets of $V$ and is not identically zero. We write $S+v$ for the translation of a set $S \subseteq V$ by a vector $v \in V$.

Definition 2.1 ([7], Definition 1, p. 221). A measure $\mu$ is said to be transverse to a Borel set $S \subset V$ if the following two conditions hold:

(i) There exists a compact set $U \subset V$ for which $0<\mu(U)<1$;

(ii) $\mu(S+v)=0$ for every $v \in V$.

Definition 2.2 ([7], Definition 2, p. 222; [1], p. 1579). A Borel set $S \subset V$ is called shy if there exists a measure transverse to $S$. More generally, a subset of $V$ is called shy if it is contained in a shy Borel set. The complement of a shy set is called a prevalent set. We say that a set is Haar ambivalent if it is neither shy nor prevalent.

Definition 2.3 ([7], p. 226). We say that "almost every" element of $V$ satisfies some given property, if the subset of $V$ on which this property holds is prevalent.

Lemma 2.4 ([7], Fact 300, p. 223). The union of a countable collection of shy sets is shy. 
Lemma 2.5 ([7], Fact 8, p. 224). If $V$ is infinite dimensional, all compact subsets of V are shy.

Lemma 2.6 ([9], Lemma 2, p. 58). Let $\mu$ be a Borel probability measure defined in complete separable metric space $V$. Then there exists a countable family of compact sets $\left(F_{k}\right)_{k \in N}$ in $V$ such that $\mu\left(V \backslash \bigcup_{k \in N} F_{k}\right)=0$.

Let $\mathbf{R}^{N}$ be a topological vector space of all real valued sequences equipped with Tychonoff metric $\rho$ defined by $\rho\left(\left(x_{k}\right)_{k \in N},\left(y_{k}\right)_{k \in N}\right)=$ $\sum_{k \in N}\left|x_{k}-y_{k}\right| / 2^{k}\left(1+\left|x_{k}-y_{k}\right|\right)$ for $\left(x_{k}\right)_{k \in N},\left(y_{k}\right)_{k \in N} \in \mathbf{R}^{N}$.

Lemma 2.7 ([16], Lemma 15.1.3, p. 202). Let $J$ be an arbitrary subset of $N$. We set

$$
A_{J}=\left\{\left(x_{i}\right)_{i \in N}: x_{i} \leq 0 \text { for } i \in J \& x_{i}>0 \text { for } i \in N \backslash J\right\} .
$$

Then the family of subsets $\Phi=\left\{A_{J}: J \subseteq N\right\}$ has the following properties:

(i) Every element of $\Phi$ is Haar ambivalent.

(ii) $A_{J_{1}} \cap A_{J_{2}}=\emptyset$ for all different $J_{1}, J_{2} \subseteq N$.

(iii) $\Phi$ is a partition of $\mathbf{R}^{N}$ such that $\operatorname{card}(\Phi)=2^{\aleph_{0}}$.

Remark 2.8. The proof of the Lemma 2.7 employs an argument stated that each Borel subset of $\mathbf{R}^{N}$ which for each compact set contains it's any translate is non-shy set.

Definition 2.9 ([10]). A sequence $\left(x_{k}\right)_{k \in N}$ of real numbers from the interval $(a, b)$ is said to be equidistributed or uniformly distributed on an interval $(a, b)$ if for any subinterval $[c, d]$ of $(a, b)$, we have

$$
\lim _{n \rightarrow \infty} n^{-1} \#\left(\left\{x_{1}, x_{2}, \cdots, x_{n}\right\} \cap[c, d]\right)=(b-a)^{-1}(d-c),
$$

where \# denotes a counting measure. 
Now, let $X$ be a compact Polish space and $\mu$ be a probability Borel measure on $X$. Let $\mathcal{R}(X)$ be a space of all bounded continuous functions defined on $X$.

Definition 2.10. A sequence $\left(x_{k}\right)_{k \in N}$ of elements of $X$ is said to be $\mu$-equidistributed or $\mu$-uniformly distributed on the $X$ if for every $f \in \mathcal{R}(X)$, we have

$$
\lim _{n \rightarrow \infty} n^{-1} \sum_{k=1}^{n} f\left(x_{k}\right)=\int_{X} f d \mu
$$

Lemma 2.11 ([10], Lemma 2.1, p. 199). Let $f \in \mathcal{R}(X)$. Then, for $\mu^{N}$-almost every sequences $\left(x_{k}\right)_{k \in N} \in X^{N}$, we have

$$
\lim _{n \rightarrow \infty} n^{-1} \sum_{k=1}^{n} f\left(x_{k}\right)=\int_{X} f d \mu .
$$

Lemma 2.12 ([10], pp. 199-201). Let $S$ be a set of all $\mu$-equidistributed sequences on $X$. Then we have $\mu^{N}(S)=1$.

Corollary 2.13 ([27], Corollary 2.3, p. 473). Let $\ell_{1}$ be a Lebesgue measure on $(0,1)$. Let $D$ be a set of all $\ell_{1}$-equidistributed sequences on $(0,1)$, Then we have $\ell_{1}^{N}(D)=1$.

Definition 2.14. Let $\mu$ be a probability Borel measure on $R$ and $F$ be its corresponding distribution function. A sequence $\left(x_{k}\right)_{k \in N}$ of elements of $R$ is said to be $\mu$-equidistributed or $\mu$-uniformly distributed on $R$ if for every interval $[a, b](-\infty \leq a<b \leq+\infty)$, we have

$$
\lim _{n \rightarrow \infty} n^{-1} \#\left([a, b] \cap\left\{x_{1}, \cdots x_{n}\right\}\right)=F(b)-F(a) .
$$

Lemma 2.15 ([27], Lemma 2.4, p. 473). Let $\left(x_{k}\right)_{k \in N}$ be $\ell_{1}$-equidistributed sequence on $(0,1), F$ be a strictly increasing continuous distribution function on $R$ and $p$ be a Borel probability measure on $R$ defined by $F$. Then $\left(F^{-1}\left(x_{k}\right)\right)_{k \in N}$ is p-equidistributed on $R$. 
Corollary 2.16 ([27], Corollary 2.4, p. 473). Let $F$ be a strictly increasing continuous distribution function on $R$ and $p_{F}$ be a Borel probability measure on $R$ defined by $F$. Then for a set $D_{F} \subset R^{N}$ of all p-equidistributed sequences on $R$, we have

(i) $D_{F}=\left\{\left(F^{-1}\left(x_{k}\right)\right)_{k \in N}:\left(x_{k}\right)_{k \in N} \in D\right\}$;

(ii) $p_{F}^{N}\left(D_{F}\right)=1$.

Lemma 2.17. Let $F_{1}$ and $F_{2}$ be two different strictly increasing continuous distribution functions on $R$ and $p_{1}$ and $p_{2}$ be Borel probability measures on $R$ defined by $F_{1}$ and $F_{2}$, respectively. Then, there does not exist a sequence of real numbers $\left(x_{k}\right)_{k \in N}$ which simultaneously is $p_{1}$-equidistributed and $p_{2}$-equidistributed.

Proof. Assume the contrary and let $\left(x_{k}\right)_{k \in N}$ be such a sequence. Since $F_{1}$ and $F_{2}$ are different there is a point $x_{0} \in R$ such that $F_{1}\left(x_{0}\right)$ $\neq F_{2}\left(x_{0}\right)$. The latter relation is not possible under our assumption, because $\left(x_{k}\right)_{k \in N}$ simultaneously is $p_{1}$-equidistributed and $p_{2}$-equidistributed, which implies

$$
F_{1}\left(x_{0}\right)=\lim _{n \rightarrow \infty} n^{-1} \#\left(\left(-\infty, x_{0}\right] \cap\left\{x_{1}, \cdots, x_{n}\right\}\right)=F_{2}\left(x_{0}\right) .
$$

Theorem 2.18. Let $F_{1}$ and $F_{2}$ be two different strictly increasing continuous distribution functions on $R$ and $p_{1}$ and $p_{2}$ be Borel probability measures on $R$ defined by $F_{1}$ and $F_{2}$, respectively. Then the measures $p_{1}^{N}$ and $p_{2}^{N}$ are orthogonal.

Proof. Let $D_{F_{1}}$ and $D_{F_{2}}$ denote $p_{1}$-equidistributed and $p_{2}$-equidistributed sequences on $R$, respectively. By Lemma 2.17 , we know that $D_{F_{1}} \cap D_{F_{2}}=\emptyset$. By Corollary 2.16 , we know that $p_{1}^{N}\left(D_{F_{1}}\right)=1$ and $p_{2}^{N}\left(D_{F_{2}}\right)=1$. 
This ends the proof of the theorem.

Definition 2.19. Let $\left\{\mu_{i}: i \in I\right\}$ be a family of probability measures defined on a measure space $(X, M)$. Let $S(X)$ be defined by

$$
S(X)=\bigcap_{i \in I} \operatorname{dom}\left(\bar{\mu}_{i}\right),
$$

where $\bar{\mu}_{i}$ denotes a usual completion of the measure $\mu_{i}$ and $\operatorname{dom}\left(\bar{\mu}_{i}\right)$ denotes the sigma-algebra of all $\bar{\mu}_{i}$-measurable subsets of $X$ for each $i \in I$. We say that the family $\left\{\mu_{i}: i \in I\right\}$ is strong separable if there exists a partition $\left\{C_{i}: i \in I\right\}$ of the space $X$ into elements of the $\sigma$-algebra $S(X)$ such that $\bar{\mu}_{i}\left(C_{i}\right)=1$ for each $i \in I$.

Definition 2.20. Let $\left\{\mu_{i}: i \in I\right\}$ be a family of probability measures defined on a measure space $(X, M)$. Let $L(I)$ denotes a minimal $\sigma$-algebra generated by all singletons of $I$ and $S(X)$ be the $\sigma$-algebra of subsets of $X$ defined by (2.7). We say that a $(S(X), L(I))$-measurable mapping $T: X \rightarrow I$ is a consistent (or well-founded) estimate of an unknown parameter $i(i \in I)$ for the family $\left\{\mu_{i}: i \in I\right\}$ if the following condition:

$$
(\forall i)\left(i \in I \rightarrow \mu_{i}\left(T^{-1}(\{i\})\right)=1\right)
$$

holds true.

Lemma 2.21 ([27], Lemma 2.5, p. 474). Let $\left\{\mu_{i}: i \in I\right\}$ be a family of probability measures defined on a measure space $(X, M)$. The following sentences are equivalent:

(i) The family of probability measures $\left\{\mu_{i}: i \in I\right\}$ is strong separable.

(ii) There exists a consistent estimate of an unknown parameter $i(i \in I)$ for the family $\left\{\mu_{i}: i \in I\right\}$. 
Now, let $X_{1}, X_{2}, \cdots$ be an infinite sampling of independent, equally distributed real-valued random variables with unknown distribution function $F$. Assume that we know only that $F$ belongs to the family of distribution functions $\left\{F_{\theta}: \theta \in \Theta\right\}$, where $\Theta$ is a non-empty set. Using these infinite sampling, we want to estimate an unknown distribution function $F$. Let $\mu_{\theta}$ denotes a Borel probability measure on the real axis $\mathbf{R}$ generated by $F_{\theta}$ for $\theta \in \Theta$. We denote by $\mu_{\theta}^{N}$ an infinite power of the measure $\mu_{\theta}$, i.e., $\mu_{\theta}^{N}=\mu_{\theta} \times \mu_{\theta} \cdots$.

The triplet $\left(\mathbf{R}^{N}, \mathcal{B}\left(\mathbf{R}^{N}\right), \mu_{\theta}^{N}\right)_{\theta \in \Theta}$ is called a statistical structure described our infinite experiment.

Definition 2.22. A Borel measurable function $T_{n}: \mathbf{R}^{n} \rightarrow \mathbf{R}(n \in N)$ is called a consistent estimator of a parameter $\theta$ (in the sense of everywhere convergence) for the family $\left(\mu_{\theta}^{N}\right)_{\theta \in \Theta}$ if the following condition:

$$
\mu_{\theta}^{N}\left(\left\{\left(x_{k}\right)_{k \in N}:\left(x_{k}\right)_{k \in N} \in \mathbf{R}^{N} \& \lim _{n \rightarrow \infty} T_{n}\left(x_{1}, \cdots, x_{n}\right)=\theta\right\}\right)=1
$$

holds true for each $\theta \in \Theta$.

Definition 2.23. A Borel measurable function $T_{n}: \mathbf{R}^{n} \rightarrow \mathbf{R}(n \in N)$ is called a consistent estimator of a parameter $\theta$ (in the sense of convergence in probability) for the family $\left(\mu_{\theta}^{N}\right)_{\theta \in \Theta}$ if for every $\epsilon>0$ and $\theta \in \Theta$ the following condition

$$
\lim _{n \rightarrow \infty} \mu_{\theta}^{N}\left(\left\{\left(x_{k}\right)_{k \in N}:\left(x_{k}\right)_{k \in N} \in \mathbf{R}^{N} \&\left|T_{n}\left(x_{1}, \cdots, x_{n}\right)-\theta\right|>\epsilon\right\}\right)=0
$$

holds true. 
Definition 2.24. A Borel measurable function $T_{n}: \mathbf{R}^{n} \rightarrow \mathbf{R}(n \in N)$ is called a consistent estimator of a parameter $\theta$ (in the sense of convergence in distribution) for the family $\left(\mu_{\theta}^{N}\right)_{\theta \in \Theta}$ if for every continuous bounded real valued function $f$ on $\mathbf{R}$ the following condition:

$$
\lim _{n \rightarrow \infty} \int_{\mathbf{R}^{N}} f\left(T_{n}\left(x_{1}, \cdots, x_{n}\right)\right) d \mu_{\theta}^{N}\left(\left(x_{k}\right)_{k \in N}\right)=f(\theta)
$$

holds.

Remark 2.25. Following [23] (see Theorem 2, p. 272), for the family $\left(\mu_{\theta}^{N}\right)_{\theta \in R}$, we have

(a) An existence of a consistent estimator of a parameter $\theta$ in the sense of everywhere convergence implies an existence of a consistent estimator of a parameter $\theta$ in the sense of convergence in probability.

(b) An existence of a consistent estimator of a parameter $\theta$ in the sense of convergence in probability implies an existence of a consistent estimator of a parameter $\theta$ in the sense of convergence in distribution.

Now, let $L(\Theta)$ be a minimal $\sigma$-algebra of subsets generated by all singletons of the set $\Theta$.

Definition 2.26. A $\left(\mathcal{B}\left(\mathbf{R}^{N}\right), L(\Theta)\right)$-measurable function $T: \mathbf{R}^{N} \rightarrow \Theta$ is called a infinite sample consistent estimate (or estimator) of a parameter $\theta$ for the family $\left(\mu_{\theta}^{N}\right)_{\theta \in \Theta}$ if the condition

$$
\mu_{\theta}^{N}\left(\left\{\left(x_{k}\right)_{k \in N}:\left(x_{k}\right)_{k \in N} \in \mathbf{R}^{N} \& T\left(\left(x_{k}\right)_{k \in N}\right)=\theta\right\}\right)=1
$$

holds true for each $\theta \in \Theta$.

Definition 2.27. An infinite sample consistent estimate $T: \mathbf{R}^{N} \rightarrow \Theta$ of a parameter $\theta$ for the family $\left(\mu_{\theta}^{N}\right)_{\theta \in \Theta}$ is called objective if $T^{-1}(\theta)$ is a Haar ambivalent set for each $\theta \in \Theta$. Otherwise, $T$ is called subjective. 
Definition 2.28. An objective infinite sample consistent estimate $T: \mathbf{R}^{N} \rightarrow \Theta$ of a parameter $\theta$ for the family $\left(\mu_{\theta}^{N}\right)_{\theta \in \Theta}$ is called strong if each $\theta_{1}, \theta_{2} \in \Theta$ there exists an isometric (with respect to Tychonoff metric) transformation $A_{\left(\theta_{1}, \theta_{2}\right)}$ of $\mathbf{R}^{N}$ such that $A_{\left(\theta_{1}, \theta_{2}\right)}\left(T^{-1}\left(\theta_{1}\right)\right) \Delta T^{-1}$ $\left(\theta_{2}\right)$ is shy.

Definition 2.29. Following [26], the family $\left(\mu_{\theta}^{N}\right)_{\theta \in \Theta}$ is called strictly separated if there exists a family $\left(Z_{\theta}\right)_{\theta \in \Theta}$ of Borel subsets of $\mathbf{R}^{N}$ such that

(i) $\mu_{\theta}^{N}\left(Z_{\theta}\right)=1$ for $\theta \in \Theta$.

(ii) $Z_{\theta_{1}} \cap Z_{\theta_{2}}=\emptyset$ for all different parameters $\theta_{1}$ and $\theta_{2}$ from $\Theta$.

(iii) $\cup_{\theta \in \Theta} Z_{\theta}=\mathbf{R}^{N}$.

Remark 2.30. Notice that an existence of an infinite sample consistent estimator of a parameter $\theta$ for the family $\left(\mu_{\theta}^{N}\right)_{\theta \in \Theta}$ implies that the family $\left(\mu_{\theta}^{N}\right)_{\theta \in \Theta}$ is strictly separated. Indeed, if we set $Z_{\theta}=\left\{\left(x_{k}\right)_{k \in N}:\left(x_{k}\right)_{k \in N} \in \mathbf{R}^{N} \& T\left(\left(x_{k}\right)_{k \in N}\right)=\theta\right\}$ for $\theta \in \Theta$, then all conditions participated in the Definition 2.29 will be satisfied.

\section{An Objective Infinite Sample Consistent Estimate of an Unknown Distribution Function}

Theorem 3.1. Let $\mathcal{F}$ be a family of distribution functions on $\mathbf{R}$ satisfying the following properties:

(i) Each element of $\mathcal{F}$ is strictly increasing and continuous.

(ii) There exists a point $x_{*}$ such that $F_{1}\left(x_{*}\right) \neq F_{2}\left(x_{*}\right)$ for each different $F_{1}, F_{2} \in \mathcal{F}$. 
Setting $\Theta=\left\{\theta=F\left(x_{*}\right): F \in \mathcal{F}\right\}$ and $F_{\theta}=F$ for $\theta=F\left(x_{*}\right)$, we get the following parametrization $\mathcal{F}=\left\{F_{\theta}: \theta \in \Theta\right\}$. We denote by $\mu_{\theta}$ a Borel probability measure in $\mathbf{R}$ defined by $F_{\theta}$ for $\theta \in \Theta$. Then a function $T_{n}: \mathbf{R}^{n} \rightarrow \mathbf{R}$, defined by

$$
T_{n}\left(x_{1}, \cdots, x_{n}\right)=\frac{\#\left(\left\{x_{1}, \cdots, x_{n}\right\} \cap\left(-\infty ; x_{*}\right]\right)}{n},
$$

for $\left(x_{1}, \cdots, x_{n}\right) \in \mathbf{R}^{n}(n \in N)$, is a consistent estimator of a parameter $\theta$ for the family $\left(\mu_{\theta}^{N}\right)_{\theta \in \Theta}$ in the sense of almost everywhere convergence.

Proof. It is clear that $T_{n}$ is Borel measurable function for $n \in N$. For $\theta \in \mathbf{R}$, we set

$$
A_{\theta}=\left\{\left(x_{k}\right)_{k \in N}:\left(x_{k}\right)_{k \in N} \text { is } \mu_{\theta} \text {-uniformly distributed on } \mathbf{R}\right\} .
$$

Following Corollary 2.16, we have $\mu_{\theta}^{N}\left(A_{0}\right)=1$ for $\theta \in \Theta$.

For $\theta \in \Theta$, we get

$$
\begin{gathered}
\mu_{\theta}^{N}\left(\left\{\left(x_{k}\right)_{k \in N} \in \mathbf{R}^{n}: \lim _{n \rightarrow \infty} T_{n}\left(x_{1}, \cdots, x_{n}\right)=\theta\right\}\right)=\mu_{\theta}^{N}\left(\left\{\left(x_{k}\right)_{k \in N} \in \mathbf{R}^{n}:\right.\right. \\
\left.\left.\lim _{n \rightarrow \infty} n^{-1} \#\left(\left\{x_{1}, \cdots, x_{n}\right\} \cap\left(-\infty ; x_{*}\right]\right)=F_{\theta}\left(x_{*}\right)\right\}\right) \geq \mu_{\theta}^{N}\left(A_{\theta}\right)=1 .
\end{gathered}
$$

The following corollaries are simple consequences of Theorem 3.1 and Remark 2.25.

Corollary 3.2. An estimator $T_{n}$ defined by (3.1) is a consistent estimator of a parameter $\theta$ for the family $\left(\mu_{\theta}^{N}\right)_{\theta \in \Theta}$ in the sense of convergence in probability.

Corollary 3.3. An estimator $T_{n}$ defined by (3.1) is a consistent estimator of a parameter $\theta$ for the family $\left(\mu_{\theta}^{N}\right)_{\theta \in \Theta}$ in the sense of convergence in distribution. 
Theorem 3.4. Let $\mathcal{F}=\left\{F_{\theta}: \theta \in \Theta\right\}$ and $\left(\mu_{\theta}^{N}\right)_{\theta \in \Theta}$ come from Theorem 3.1. Let $\theta_{0} \in \Theta$ and define an estimate $T_{\theta_{0}}^{(1)}: \mathbf{R}^{n} \rightarrow \Theta$ as follows: $T_{\theta_{0}}^{(1)}\left(\left(x_{k}\right)_{k \in N}\right)=\varlimsup \widetilde{\lim } \widetilde{T_{n}}\left(\left(x_{k}\right)_{k \in N}\right)$ if $\widetilde{\lim } \widetilde{T_{n}}\left(\left(x_{k}\right)_{k \in N}\right) \in \Theta \backslash\left\{\theta_{0}\right\}$ and $T_{\theta_{0}}^{(1)}\left(\left(x_{k}\right)_{k \in N}\right)=\theta_{0}$, otherwise, where $\widetilde{\lim } \widetilde{T_{n}}=\inf _{n} \sup _{m \geq n} \widetilde{T_{m}}$ and

$$
\widetilde{T_{n}}\left(\left(x_{k}\right)_{k \in N}\right)=n^{-1} \#\left(\left\{x_{1}, \cdots, x_{n}\right\} \cap\left(-\infty ; x_{*}\right)\right),
$$

for $\left(x_{k}\right)_{k \in N} \in \mathbf{R}^{N}$. Then $T_{\theta_{0}}^{(1)}$ is an objective infinite sample consistent estimator of a parameter $\theta$ for the family $\left(\mu_{\theta}^{N}\right)_{\theta \in \Theta}$.

Proof. Following [23] (see p. 189), the function $\varlimsup \widetilde{\lim } \widetilde{T_{n}}$ is Borel measurable which implies that the function $\widetilde{\lim \widetilde{T_{n}}}$ is $\left(\mathcal{B}\left(\mathbf{R}^{N}\right), L(\Theta)\right)$ measurable. Following Corollary 2.16, we have $\mu_{\theta}^{N}\left(A_{\theta}\right)=1$ for $\theta \in \Theta$, where $A_{\theta}$ is defined by (3.2). Hence, we get

$$
\begin{aligned}
& \mu_{\theta}^{N}\left(\left\{\left(x_{k}\right)_{k \in N} \in \mathbf{R}^{N}: T_{\theta_{0}}^{(1)}\left(x_{k}\right)_{k \in N}=\theta\right\}\right) \\
& \geq \mu_{\theta}^{N}\left(\left\{\left(x_{k}\right)_{k \in N} \in \mathbf{R}^{N}: \varlimsup \widetilde{\lim } \widetilde{T_{n}}\left(x_{k}\right)_{k \in N}=\theta\right\}\right) \\
& \geq \mu_{\theta}^{N}\left(\left\{\left(x_{k}\right)_{k \in N} \in \mathbf{R}^{N}: \varlimsup \overline{\lim } \widetilde{T_{n}}\left(x_{k}\right)_{k \in N}\right.\right.
\end{aligned}
$$

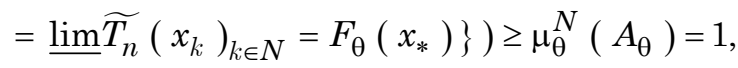

for $\theta \in \Theta$. Thus, we have proved that the estimator $T_{\theta_{0}}^{(1)}$ is an infinite sample consistent estimator of a parameter $\theta$ for the family $\left(\mu_{\theta}^{N}\right)_{\theta \in \Theta}$.

Now, let us show that $\mathbf{T}_{\theta_{0}}^{(1)}$ is an objective infinite sample consistent estimator of a parameter $\theta$ for the family $\left(\mu_{\theta}^{N}\right)_{\theta \in \Theta}$. 
Let us show that $B(\theta):=\left(\mathbf{T}_{\theta_{0}}^{(1)}\right)^{-1}(\theta)$ is a Haar ambivalent set for each $\theta \in \Theta$.

Let $\left(x_{k}\right)_{k \in N}$ be $\mu_{\theta}$-uniformly distributed sequence on $\mathbf{R}$. Then we get

$$
\lim _{n \rightarrow \infty} n^{-1} \#\left(\left\{x_{1}, \cdots, x_{n}\right\} \cap\left(-\infty ; x_{*}\right]\right)=\theta .
$$

Let us consider a set

$$
C(\theta)=\left\{\left(y_{k}\right)_{k \in N}: y_{k} \leq x_{k} \text { if } x_{k} \leq x_{*} \& y_{k}>x_{k} \text { if } x_{k}>x_{*}\right\} .
$$

Setting $J=\left\{k: x_{k} \leq x_{*}\right\}$, we claim that $C(\theta)-\left(x_{k}\right)_{k \in N}=A_{J}$, where $A_{J}$ comes from Lemma 2.7. Since any translate of Haar ambivalent set is again Haar ambivalent set, we claim that $C(\theta)$ is Haar ambivalent set. A set $B(\theta)$ which contains the Haar ambivalent set $C(\theta)$ is non-shy. Since $\theta \in \Theta$ was taken arbitrary we deduce that each $B_{\theta}$ is Haar ambivalent set. The latter relation means that the estimator $\mathbf{T}_{\theta_{0}}^{(1)}$ is an objective infinite sample consistent estimator of a parameter $\theta$ for the family $\left(\mu_{\theta}^{N}\right)_{\theta \in \Theta}$.

Theorem 3.5. Let $\mathcal{F}=\left\{F_{\theta}: \theta \in \Theta\right\}$ and $\left(\mu_{\theta}^{N}\right)_{\theta \in \Theta}$ come from Theorem 3.1. Let fix $\theta_{0} \in \Theta$ and define an estimate $T_{\theta_{0}}^{(2)}: \mathbf{R}^{N} \rightarrow \Theta$ as

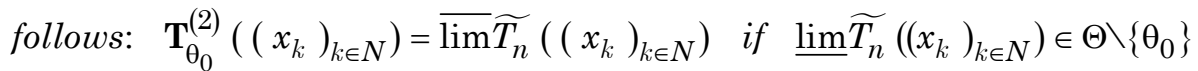

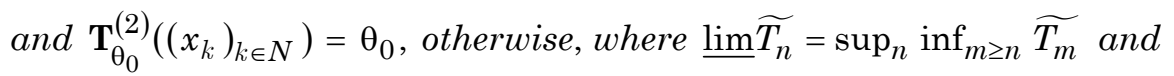

$$
\widetilde{T_{n}}\left(\left(x_{k}\right)_{k \in N}\right)=n^{-1} \#\left(\left\{x_{1}, \cdots, x_{n}\right\} \cap\left(-\infty ; x_{*}\right]\right),
$$

for $\left(x_{k}\right)_{k \in N} \in \mathbf{R}^{N}$. Then $\mathbf{T}_{\theta_{0}}^{(2)}$ is an objective infinite sample consistent estimator of a parameter $\theta$ for the family $\left(\mu_{\theta}^{N}\right)_{\theta \in \Theta}$. 
Proof. Following [23] (see p. 189), the function $\underline{\lim \widetilde{T_{n}}}$ is Borel measurable which implies that the function $\varlimsup \widetilde{\lim } \widetilde{T_{n}}$ is $\left(\mathcal{B}\left(\mathbf{R}^{n}\right), L(\Theta)\right)$ measurable. Following Corollary 2.16, we have $\mu_{\theta}^{N}\left(A_{\theta}\right)=1$ for $\theta \in \Theta$, where $A_{\theta}$ is defined by (3.2). Hence, we get

$$
\begin{aligned}
& \mu_{\theta}^{N}\left(\left\{\left(x_{k}\right)_{k \in N} \in \mathbf{R}^{N}: \mathbf{T}_{\theta_{0}}^{(2)}\left(x_{k}\right)_{k \in N}=\theta\right\}\right) \\
& \geq \mu_{\theta}^{N}\left(\left\{\left(x_{k}\right)_{k \in N} \in \mathbf{R}^{N}: \underline{\lim } \widetilde{T_{n}}\left(x_{k}\right)_{k \in N}=\theta\right\}\right) \\
& \geq \mu_{\theta}^{N}\left(\left\{\left(x_{k}\right)_{k \in N} \in \mathbf{R}^{N}: \widetilde{\lim } \widetilde{T_{n}}\left(x_{k}\right)_{k \in N}\right.\right. \\
& \left.\left.=\underline{\lim } \widetilde{T_{n}}\left(x_{k}\right)_{k \in N}=F_{\theta}\left(x_{*}\right)\right\}\right) \geq \mu_{\theta}^{N}\left(A_{\theta}\right)=1,
\end{aligned}
$$

for $\theta \in \Theta$.

Thus, we have proved that the estimator $\mathbf{T}_{\theta_{0}}^{(2)}$ is an infinite sample consistent estimators of a parameter $\theta$ for the family $\left(\mu_{\theta}^{N}\right)_{\theta \in \Theta}$.

Now, let us show that $\mathbf{T}_{\theta_{0}}^{(2)}$ is an objective infinite sample consistent estimator of a parameter $\theta$ for the family $\left(\mu_{\theta}^{N}\right)_{\theta \in \Theta}$.

Let us show that $B(\theta)=\left(\mathbf{T}_{\theta_{0}}^{(2)}\right)^{-1}(\theta)$ is Haar ambivalent set for each $\theta \in \Theta$.

Let $\left(x_{k}\right)_{k \in N}$ be $\mu_{\theta}$-uniformly distributed sequence. Then we get

$$
\lim _{n \rightarrow \infty} n^{-1} \#\left(\left\{x_{1}, \cdots, x_{n}\right\} \cap\left(-\infty ; x_{*}\right]\right)=\theta .
$$

Let consider a set

$$
\begin{array}{r}
C(\theta)=\left\{\left(y_{k}\right)_{k \in N}:\left(y_{k}\right)_{k \in N} \in \mathbf{R}^{N} \& y_{k} \leq x_{k} \text { if } x_{k} \leq x_{*}\right. \\
\left.\& y_{k}>x_{k} \text { if } x_{k}>x_{*}\right\} .
\end{array}
$$


Setting $J=\left\{k: x_{k} \leq x_{*}\right\}$, we deduce that $C(\theta)-\left(x_{k}\right)_{k \in N}=A_{J}$, where $A_{J}$ comes from Lemma 2.7. Since any translate of Haar ambivalent set is again Haar ambivalent set, we claim that $C(\theta)$ is Haar ambivalent set. A set $B(\theta)$ which contains the Haar ambivalent set $C(\theta)$ is non-shy. Since $\theta \in \Theta$ was taken arbitrary we deduce that each $B_{\theta}$ is Haar ambivalent set. The latter relation means that the estimator $\mathbf{T}_{\theta_{0}}^{(2)}$ is an objective infinite sample consistent estimator of a parameter $\theta$ for the family $\left(\mu_{\theta}^{N}\right)_{\theta \in \Theta}$.

Remark 3.6. It can be shown that Theorems 3.4 and 3.5 extend the recent result obtained in [20] (see Theorem 3.1). Indeed, let consider the linear one-dimensional stochastic system

$$
\left(\xi_{k}\right)_{k \in N}=\left(\theta_{k}\right)_{k \in N}+\left(\Delta_{k}\right)_{k \in N}
$$

where $\left(\theta_{k}\right)_{k \in N} \in \mathbf{R}^{N}$ is a sequence of useful signals, $\left(\Delta_{k}\right)_{k \in N}$ is sequence of independent identically distributed random variables (the so-called generalized "white noise") defined on some probability space $(\Omega, \mathcal{F}, P)$ and $\left(\xi_{k}\right)_{k \in N}$ is a sequence of transformed signals. Let $\mu$ be a Borel probability measure on $\mathbf{R}$ defined by a random variable $\Delta_{1}$. Then the $N$-power of the measure $\mu$ denoted by $\mu^{N}$ coincides with the Borel probability measure on $\mathbf{R}^{N}$ defined by the generalized "white noise", i.e., $(\forall X)\left(X \in \mathcal{B}\left(\mathbf{R}^{N}\right) \rightarrow \mu^{N}(X)=P\left(\left\{\omega: \omega \in \Omega \&\left(\Delta_{k}(\omega)\right)_{k \in N} \in X\right\}\right)\right)$, (3.13) where $\mathcal{B}\left(\mathbf{R}^{N}\right)$ is the Borel $\sigma$-algebra of subsets of $\mathbf{R}^{N}$.

Following [26], a general decision in the information transmission theory is that the Borel probability measure $\lambda$, defined by the sequence of transformed signals $\left(\xi_{k}\right)_{k \in N}$ coincides with $\left(\mu^{N}\right)_{\theta_{0}}$ for some $\theta_{0} \in \Theta$ provided that 


$$
\left(\exists \theta_{0}\right)\left(\theta_{0} \in \Theta \rightarrow(\forall X)\left(X \in \mathcal{B}\left(\mathbf{R}^{N}\right) \rightarrow \lambda(X)=\left(\mu^{N}\right)_{\theta_{0}}(X)\right)\right),
$$

where $\left(\mu^{N}\right)_{\theta_{0}}(X)=\mu^{N}\left(X-\theta_{0}\right)$ for $X \in \mathcal{B}\left(\mathbf{R}^{N}\right)$.

In [27] has been considered a particular case of the above model (3.12) for which

$$
\left(\theta_{k}\right)_{k \in N} \in\{(\theta, \theta, \cdots): \theta \in \mathbf{R}\}
$$

For $\theta \in \mathbf{R}$, a measure $\mu_{\theta}^{N}$ defined by

$$
\mu_{\theta}^{N}=\mu_{\theta} \times \mu_{\theta} \times \cdots
$$

where $\mu_{\theta}$ is a $\theta$-shift of $\mu\left(\right.$ i.e., $\mu_{\theta}(X)=\mu(X-\theta)$ for $(X \in \mathcal{B}(\mathbf{R}))$, is called the $N$-power of the $\theta$-shift of $\mu$ on $\mathbf{R}$.

Let denote by $F_{\theta}$ a distribution function defined by $\mu_{\theta}$ for $\theta \in \Theta$. Notice that the family $\mathcal{F}=\left\{F_{\theta}: \theta \in \Theta\right\}$ satisfies all conditions participated in Theorem 3.1. Indeed, under $x_{*}$ we can take the zero of the real axis. Then following Theorems 3.4 and 3.5, estimators $T_{\theta_{0}}^{(1)}$ and $T_{\theta_{0}}^{(2)}$ are objective infinite sample consistent estimators of a useful signal $\theta$ in the linear one-dimensional stochastic system (3.12). Notice that these estimators exactly coincide with estimators constructed in [20] (see Theorem 3.1).

Theorem 3.7. Let $\mathcal{F}$ be a family of all strictly increasing and continuous distribution functions in $\mathbf{R}$ and $p_{F}$ be a Borel probability measure on $\mathbf{R}$ defined by $F$ for each $F \in \mathcal{F}$. Then the family of Borel probability measures $\left\{p_{F}^{N}: F \in \mathcal{F}\right\}$ is strong separable.

Proof. We denote by $D_{F}$ the set of all $p_{F}$-equidistributed sequences on $\boldsymbol{R}$ for each $F \in \mathcal{F}$. By Lemma 2.17, we know that $D_{F_{1}} \cap D_{F_{2}}=\emptyset$ for each different $F_{1}, F_{2} \in \mathcal{F}$. By Corollary 2.16, we know that 
$p_{F}^{N}\left(D_{F}\right)=1$ for each $F \in \mathcal{F}$. Let fix $F_{0} \in \mathcal{F}$ and define a family $\left(C_{F}\right)_{F \in \mathcal{F}}$ of subsets of $\mathbf{R}^{N}$ as follows: $C_{F}=D_{F}$ for $F \in \mathcal{F} \backslash\left\{F_{0}\right\}$ and $C_{F_{0}}=\mathbf{R}^{N} \backslash \bigcup_{F \in \mathcal{F} \backslash\left\{F_{0}\right\}} D_{F}$. Notice that since $D_{F}$ is a Borel subset of $\mathbf{R}^{N}$ for each $F \in \mathcal{F}$, we claim that $C_{F} \in S\left(\mathbf{R}^{N}\right)$ for each $F \in \mathcal{F} \backslash\left\{F_{0}\right\}$, where $S\left(\mathbf{R}^{N}\right)$ comes from Definition 2.19. Since $\overline{p_{F}^{N}}\left(\mathbf{R}^{N} \backslash \bigcup_{F \in \mathcal{F}} D_{F}\right)=0$ for each $F \in \mathcal{F}$, we deduce that $\mathbf{R}^{N} \backslash \bigcup_{F \in \mathcal{F}} D_{F} \in \bigcap_{F \in \mathcal{F}} \operatorname{dom}\left(\overline{p_{F}^{N}}\right)=S\left(\mathbf{R}^{N}\right)$. Since $S\left(\mathbf{R}^{N}\right)$ is the $\sigma$-algebra we claim that $C_{F_{0}} \in S\left(\mathbf{R}^{N}\right)$, because $\overline{p_{F}^{N}}\left(\mathbf{R}^{N} \backslash \bigcup_{F \in \mathcal{F}} D_{F}\right)=0$ for each $F \in \mathcal{F} \quad$ (equivalently, $\left.\mathbf{R}^{N} \backslash \bigcup_{F \in \mathcal{F}} D_{F} \in S\left(\mathbf{R}^{N}\right)\right)$, and

$$
C_{F_{0}}=\mathbf{R}^{N} \backslash \bigcup_{F \in \mathcal{F} \backslash\left\{F_{0}\right\}} D_{F}=\left(\mathbf{R}^{N} \backslash \bigcup_{F \in \mathcal{F}} D_{F}\right) \cup D_{F_{0}} .
$$

This ends the proof of the theorem.

Remark 3.8. By virtue the results of Lemma 2.21 and Theorem 3.7, we get that there exists a consistent estimate of an unknown distribution function $F(F \in \mathcal{F})$ for the family of Borel probability measures $\left\{p_{F}^{N}: F \in \mathcal{F}\right\}$, where $\mathcal{F}$ comes from Theorem 3.7. This estimate $T: \mathbf{R}^{N} \rightarrow \mathcal{F}$ is defined by $: T\left(\left(x_{k}\right)_{k \in N}\right)=F$ if $\left(x_{k}\right)_{k \in N} \in C_{F}$, where the family $\left(C_{F}\right)_{F \in \mathcal{F}}$ of subsets of $\mathbf{R}^{N}$ also comes from Theorem 3.7. Notice that this result extends the main result established in [27] (see Lemma 2.6, p. 476).

At end of this section, we state the following:

Problem 3.1. Let $\mathcal{F}$ be a family of all strictly increasing and continuous distribution functions on $\mathbf{R}$ and $p_{F}$ be a Borel probability measure in $\mathbf{R}$ defined by $F$ for each $F \in \mathcal{F}$. Does there exist an objective infinite sample consistent estimate of an unknown distribution function $F$ for the family of Borel probability measures $\left\{p_{F}^{N}: F \in \mathcal{F}\right\}$ ? 


\section{An Effective Construction of the Strong Objective infinite Sample Consistent Estimate of a Useful Signal in the Linear one-Dimensional Stochastic Model}

In [18], the examples of objective and strong objective infinite sample consistent estimates ([18], $T^{\star}$ (p. 63), $T^{\circ}$ (p. 67)) of a useful signal in the linear one-dimensional stochastic model were constructed by using the axiom of choice and a certain partition of the non-locally compact abelian Polish group $R^{N}$ constructed in [17].

In this section, in the same model, we present an effective example of the strong objective infinite sample consistent estimate of a useful signal constructed in [19].

For each real number $a \in R$, we denote by $\{a\}$ its fractal part in the decimal system.

Theorem 4.1. Let consider the linear one-dimensional stochastic model (3.12), for which "white noise" has a infinite absolute moment of the first order and its moment of the first order is equal to zero. Suppose that the Borel probability measure $\lambda$, defined by the sequence of transformed signals $\left(\xi_{k}\right)_{k \in N}$ coincides with $\left(\mu_{\theta_{0}}^{N}\right)$ for some $\theta_{0} \in[0,1]$.

Let $T: R^{N} \rightarrow[0,1]$ be defined by: $T\left(\left(x_{k}\right)_{k \in N}\right)=\left\{\lim _{n \rightarrow \infty} \frac{\sum_{k=1}^{n} x_{k}}{n}\right\}$ if $\lim _{n \rightarrow \infty} \frac{\sum_{k=1}^{n} x_{k}}{n} \neq 1 ; T\left(\left(x_{k}\right)_{k \in N}\right)=1$ if $\lim _{n \rightarrow \infty} \frac{\sum_{k=1}^{n} x_{k}}{n}=1$; and $T\left(\left(x_{k}\right)_{k \in N}\right)=\sum_{k \in N} \frac{\chi_{(0,+\infty)}\left(x_{k}\right)}{2^{k}}$, otherwise, where $\chi_{(0,+\infty)}(\cdot)$ denotes an indicator function of the set $(0,+\infty)$ defined on the real axis $R$. Then $T$ is a strong objective infinite sample consistent estimate of the parameter $\theta$ for the statistical structure $\left(R^{N}, \mathcal{B}\left(R^{N}\right), \mu_{\theta}^{N}\right)_{\theta \in \Theta}$ describing the linear onedimensional stochastic system (3.12). 
Proof. Step 1. We have to show that $T$ is an infinite sample consistent estimate of the parameter $\theta$ for the statistical structure $\left(R^{N}, \mathcal{B}\left(R^{N}\right), \mu_{\theta}^{N}\right)_{\theta \in \Theta}$ and $T^{-1}(\theta)$ is a Haar ambivalent set for each $\theta=\sum_{k=1}^{\infty} \frac{\theta_{k}}{2^{k}} \in \Theta$, where $\sum_{k=1}^{\infty} \frac{\theta_{k}}{2^{k}}$ is representation of the number $\theta$ in the binary system.

Indeed, we have

$$
(\forall \theta)\left(\theta \in(0,1) \rightarrow T^{-1}(\theta)=\left(B_{H(\theta)} \backslash S\right) \cup \cup_{z \in Z} S_{\theta+z}\right),
$$

where $H(\theta)=\left\{k: k \in N \& \theta_{k}=1\right\}, B_{H(\theta)}=\theta-A_{H(\theta)}, A_{H(\theta)}$ comes from Lemma 2.7,

$$
S=\left\{\left(x_{k}\right)_{k \in N} \in \mathbf{R}^{N}: \text { exists a finite limit } \lim _{n \rightarrow \infty} \frac{\sum_{k=1}^{n} x_{k}}{n}\right\},
$$

and

$$
S_{\theta+z}=\left\{\left(x_{k}\right)_{k \in N} \in \mathbf{R}^{N}: \lim _{n \rightarrow \infty} \frac{\sum_{k=1}^{n} x_{k}}{n}=\theta+z\right\},
$$

for each $\theta \in \Theta$ and $z \in Z$. Notice that the set $S$ like $\bigcup_{z \in Z} S_{\theta+z}$ is Borel shy set (see [18], Lemma 4.14, p. 60). Taking into account this fact, the results of Lemmas 2.4 and 2.7 invariance of Haar ambivalent sets under translations and symmetric transformation and the simple statement that difference of non-shy and shy sets is non-shy, we deduce that $T^{-1}(\theta)$ is a Borel measurable Haar ambivalent sets for each $\theta \in \Theta$.

Notice that

$$
T^{-1}(1)=\left(B_{H(1)} \backslash S\right) \cup S_{1}=\left(B_{N} \backslash S\right) \cup S_{1},
$$

and

$$
T^{-1}(0)=\left(B_{H(0)} \backslash S\right) \cup \bigcup_{z \in Z \backslash\{1\}} S_{0+z}=\left(B_{\emptyset} \backslash S\right) \cup \bigcup_{z \in Z \backslash\{1\}} S_{0+z},
$$

which also are Borel measurable Haar ambivalent sets. 
Now, it is not hard to show that $T$ is $\left(\mathcal{B}\left(\mathbf{R}^{N}\right), L(\Theta)\right)$-measurable because the class $\mathcal{B}\left(\mathbf{R}^{N}\right)$ is closed under countable family of settheoretical operations and each element of $L(\Theta)$ is countable or co-countable in the interval $\theta=[0,1]$. Since $S_{\theta} \subseteq T^{-1}(\theta)$ for $\theta \in \Theta$, we deduce that $\mu_{\theta}\left(T^{-1}(\theta)\right)=1$. The later relation means that $T$ is an infinite sample consistent estimate of a parameter $\theta$.

Step 2. Let us show that for each different $\theta_{1}, \theta_{2} \in[0,1]$ there exists an isometric (with respect to Tychonoff metric) transformation $A_{\left(\theta_{1}, \theta_{2}\right)}$ such that

$$
A_{\left(\theta_{1}, \theta_{2}\right)}\left(T^{-1}\left(\theta_{1}\right)\right) \Delta T^{-1}\left(\theta_{2}\right)
$$

is shy.

We define $A_{\left(\theta_{1}, \theta_{2}\right)}$ as follows: for $\left(x_{k}\right)_{k \in N} \in R^{N}$ we put $A_{\left(\theta_{1}, \theta_{2}\right)}\left(\left(x_{k}\right)_{k \in N}\right)=\left(y_{k}\right)_{k \in N}$, where $\quad y_{k}=-x_{k} \quad$ if $\quad k \in H\left(\theta_{1}\right) \Delta H\left(\theta_{2}\right)$ $\left(:=\left(H\left(\theta_{1}\right) \backslash H\left(\theta_{2}\right)\right) \cup\left(H\left(\theta_{2}\right) \backslash H\left(\theta_{1}\right)\right)\right.$ and $y_{k}=x_{k}$, otherwise. It is obvious that $A_{\left(\theta_{1}, \theta_{2}\right)}$ is isometric (with respect to Tychonoff metric) transformation of the $R^{N}$.

Notice that

$$
A_{\left(\theta_{1}, \theta_{2}\right)}\left(T^{-1}\left(\theta_{1}\right)\right) \Delta T^{-1}\left(\theta_{2}\right) \subseteq \bigcup_{k \in N}\{0\}_{k} \times R^{N \backslash\{k\}} \cup S .
$$

Since both sets $\bigcup_{k \in N}\{0\}_{k} \times R^{N \backslash\{k\}}$ and $S$ are shy, by Lemmas 2.4 and Definition 2.2, we claim that the set

$$
A_{\left(\theta_{1}, \theta_{2}\right)}\left(T^{-1}\left(\theta_{1}\right)\right) \Delta T^{-1}\left(\theta_{2}\right)
$$

is also shy.

This ends the proof of the theorem. 


\section{On Infinite Sample Consistent Estimates of an Unknown Probability Density Function}

Let $X_{1}, X_{2}, \cdots$ be independent identically distributed real-valued random variables having a common probability density function $f$. After a so-called kernel class of estimates $f_{n}$ of $f$ based on $X_{1}, X_{2}, \cdots, X_{n}$ was introduced by Rosenblatt [21], various convergence properties of these estimates have been studied. The stronger result in this direction was due to Nadaraya [13] who proved that if $f$ is uniformly continuous, then for a large class of kernels the estimates $f_{n}$ converges uniformly on the real line to $f$ with probability one. In [22] has been shown that the above assumptions on $f$ are necessary for this type of convergence. That is, if $f_{n}$ converges uniformly to a function $g$ with probability one, then $g$ must be uniformly continuous and the distribution $F$ from which we are sampling must be absolutely continuous with $F^{\prime}(x)=g(x)$ everywhere. When in addition to the mentioned above, it is assumed that $f$ and its first $r+1$ derivatives are bounded, it is possible to show that how to construct estimates $f_{n}$ such that $f_{n}^{(s)}$ converges uniformly to $f^{(s)}$ as a given rate with probability one for $s=0, \cdots, r$. Let $f_{n}(x)$ be a kernel estimate based on $X_{1}, X_{2}, \cdots, X_{n}$ from $F$ as given in [21], that is,

$$
f_{n}(x)=\left(n a_{n}\right)^{-1} \sum_{i=1}^{n} k\left(\frac{X_{i}-x}{a_{n}}\right)
$$

where $\left(a_{n}\right)_{n \in N}$ is a sequence of positive numbers converging to zero and $k$ is a probability density function such that $\int_{-\infty}^{+\infty}|x| k(x) d x$ is finite and $k^{(s)}$ is continuous function of bounded variation for $s=0, \cdots, r$. The density function of the standard normal, for example, satisfies all these conditions.

In the sequel, we need the following wonderful statement: 
Lemma 5.1 ([22], Theorem 3.11, p. 1194). A necessary and sufficient condition for

$$
\lim _{n \rightarrow \infty} \sup _{x \in \mathbf{R}}\left|f_{n}(x)-g(x)\right|=0,
$$

with probability one for a function $g$ is that $g$ be the uniformly continuous derivative of $F$.

Let $X_{1}, X_{2}, \cdots$ be independent and identically distributed realvalued random variables with an unknown probability density function $f$. Assume that we know that $f$ belongs to the class of probability density function $\mathcal{S C}$ each element of which is uniformly continuous.

Let denote by $\ell^{\infty}(R)$ an infinite-dimensional non-separable Banach space of all bounded real-valued functions on $\mathbf{R}$ equipped with norm $\|\cdot\|_{\infty}$ defined by

$$
\|h\|_{\infty}=\sup _{x \in \mathbf{R}}|h(x)|
$$

for all $h \in \ell^{\infty}(R)$. We say that $\left(\ell^{\infty}(R)\right) \lim _{n \rightarrow \infty} h_{n}=h_{0}$ if $\lim _{n \rightarrow \infty} \| h_{n}-$ $h_{0} \|_{\infty}=0$.

Theorem 5.2. Let $\phi$ denotes a normal density function. We set $\Theta=\mathcal{S C}$. Let $\mu_{\theta}$ be a Borel probability measure on $\mathbf{R}$ with probability density function $\theta \in \Theta$. Let fix $\theta_{0} \in \Theta$. For each $\left(x_{i}\right)_{i \in N}$, we set $T_{\mathcal{S C}}\left(\left(x_{i}\right)_{i \in N}\right)=\left(\ell^{\infty}(R)\right) \lim _{n \rightarrow \infty} f_{n}$ if this limit exists and is in $\Theta \backslash\left\{\theta_{0}\right\}$, and $T_{\mathcal{S C}}\left(\left(x_{i}\right)_{i \in N}\right)=\theta_{0}$, otherwise. Then $T_{\mathcal{S C}}$ is a consistent infinitesample estimate of an unknown parameter $\theta$ for the family $\left(\mu_{\theta}^{N}\right)_{\theta \in \Theta}$.

Proof. By Lemma 5.1, for each $\theta \in \Theta$, we have

$$
\begin{aligned}
& \mu_{\theta}^{N}\left(\left\{\left(x_{i}\right)_{i \in N} \in \mathbf{R}^{N}: T_{\mathcal{S C}}\left(\left(x_{i}\right)_{i \in N}\right)=\theta\right\}\right) \\
& \quad \geq \mu_{\theta}^{N}\left(\left\{\left(x_{i}\right)_{i \in N} \in \mathbf{R}^{N}:\left(\ell^{\infty}(R)\right) \lim _{n \rightarrow \infty} f_{n}=\theta\right\}\right) \\
& \quad=\mu_{\theta}^{N}\left(\left\{\left(x_{i}\right)_{i \in N} \in \mathbf{R}^{N}: \lim _{n \rightarrow \infty}\left\|f_{n}-\theta\right\|_{\infty}=0\right\}\right)=1 .
\end{aligned}
$$


This ends the proof of theorem.

Concerning with Theorem 5.2, we state the following problems:

Problem 5.1. Let $T_{\mathcal{S C}}$ comes from the Theorem 5.2. Is $T_{\mathcal{S C}}$ an objective infinite sample consistent estimate of the parameter $\theta$ for the family $\left(\mu_{\theta}^{N}\right)_{\theta \in \Theta}$ ?

Problem 5.2. Let the statistical structure $\left\{\left(\mathbf{R}^{N}, \mathcal{B}\left(\mathbf{R}^{N}\right), \mu_{\theta}^{N}\right): \theta \in \Theta\right\}$ comes from the Theorem 5.2. Does there exist an objective (or strong objective) infinite sample consistent estimate of the parameter $\theta$ for the family $\left(\mu_{\theta}^{N}\right)_{\theta \in \Theta}$ ?

Let $X_{1}, X_{2}, \cdots$ be independent and identically distributed real-valued random variables with positive continuous probability density function $f$. Assume that we know that $f$ belongs to the separated class $\mathcal{A}$ of positive continuous probability densities provided that there is a point $x_{*}$ such that $g_{1}\left(x_{*}\right) \neq g_{2}\left(x_{*}\right)$ for each $g_{1}, g_{2} \in \mathcal{A}$. Suppose that we have an infinite sample $\left(x_{k}\right)_{k \in N}$ and we want to estimate an unknown probability density function. Setting $\Theta=\left\{\theta=g\left(x_{*}\right): g \in \mathcal{A}\right\}$, we can parameterise the family $\mathcal{A}$ as follows: $\mathcal{A}=\left\{f_{\theta}: \theta \in \Theta\right\}$, where $f_{\theta}$ is such a unique element $f$ from the family $\mathcal{A}$ for which $f\left(x_{*}\right)=\theta$. Let $\mu_{\theta}$ be a Borel probability measure defined by the probability density function $f_{\theta}$ for each $\theta \in \Theta$. It is obvious that $\left\{\left(\mathbf{R}^{N}, \mathcal{B}\left(\mathbf{R}^{N}\right), \mu_{\theta}^{N}\right): \theta \in \Theta\right\}$ will be the statistical structure described our experiment.

Theorem 5.3. Let $\left(h_{m}\right)_{m \in N}$ be a sequence of a strictly decreasing sequence of positive numbers tending to zero. Let $\theta_{0} \in \Theta$. For each $\left(x_{k}\right)_{k \in N} \in \mathbf{R}^{N}$, we put

$$
T\left(\left(x_{k}\right)_{k \in N}\right)=\lim _{m \rightarrow \infty} \lim _{n \rightarrow \infty} \frac{\#\left(\left\{x_{1}, \cdots, x_{n}\right\} \cap\left[x_{*}-h_{m}, x_{*}+h_{m}\right]\right)}{2 n h_{m}},
$$


if this repeated limit exists and belongs to the set $\Theta \backslash\{\theta\}$, and $T\left(\left(x_{k}\right)_{k \in N}\right)=\theta_{0}$, otherwise. Then $T$ is an infinite sample consistent estimate of the parameter $\theta$ for the family $\left(\mu_{\theta}^{N}\right)_{\theta \in \Theta}$.

Proof. For each $\theta \in \Theta$, we put

$$
A_{\theta}=\left\{\left(x_{k}\right)_{k \in N}:\left(x_{k}\right)_{k \in N} \in \mathbf{R}^{N} \&\left(x_{k}\right)_{k \in N} \text { is } \mu_{\theta} \text {-equidistributed }\right\} .
$$

By Corollary 2.16, we know that $\mu_{\theta}^{N}\left(A_{\theta}\right)=1$ for each $\theta \in \Theta$.

For each $\theta \in \Theta$, we have

$$
\begin{aligned}
\mu_{\theta}^{N}\left(T^{-1}(\theta)\right) & =\mu_{\theta}^{N}\left(\left\{\left(x_{k}\right)_{k \in N} \in \mathbf{R}^{N}: T\left(\left(x_{k}\right)_{k \in N}\right)=\theta\right\}\right) \\
& \geq \mu_{\theta}^{N}\left(\left\{\left(x_{k}\right)_{k \in N} \in A_{\theta}: T\left(\left(x_{k}\right)_{k \in N}\right)=\theta\right\}\right) \\
& =\mu_{\theta}^{N}\left(\left\{\left(x_{k}\right)_{k \in N} \in A_{\theta}: \lim _{m \rightarrow \infty} \frac{F_{\theta}\left(x_{*}+h_{m}\right)-F_{\theta}\left(x_{*}-h_{m}\right)}{2 h_{m}}=\theta\right\}\right) \\
& =\mu_{\theta}^{N}\left(\left\{\left(x_{k}\right)_{k \in N} \in A_{\theta}: \lim _{m \rightarrow \infty} \frac{\int_{x_{*}-h_{m}}^{x_{*}+h_{m}} f_{\theta}(x) d x}{2 h_{m}}=\theta\right\}\right) \\
& =\mu_{\theta}^{N}\left(\left\{\left(x_{k}\right)_{k \in N} \in A_{\theta}: f_{\theta}\left(x^{*}\right)=\theta\right\}\right)=\mu_{\theta}^{N}\left(A_{\theta}\right)=1 .
\end{aligned}
$$

Concerning with Theorem 5.3, we state the following:

Problem 5.3. Let $T$ comes from the Theorem 5.3. Is $T$ an objective infinite sample consistent estimate of the parameter $\theta$ for the family $\left(\mu_{\theta}^{N}\right)_{\theta \in \Theta} ?$

Problem 5.4. Let the statistical structure $\left\{\left(\mathbf{R}^{N}, \mathcal{B}\left(\mathbf{R}^{N}\right), \mu_{\theta}^{N}\right): \theta \in \Theta\right\}$ comes from the Theorem 5.3. Does there exist an objective (or strong objective) infinite sample consistent estimate of the parameter $\theta$ for the family $\left(\mu_{\theta}^{N}\right)_{\theta \in \Theta}$ ? 
Example 5.4. Let $X_{1}, X_{2}, \cdots$ be independent normally distributed real-valued random variables with parameters $(a, \sigma)$ where a is a mean and $\sigma$ is a standard deviation. Suppose that we know the mean a and want to estimate an unknown standard deviation $\sigma$ by an infinite sample $\left(x_{k}\right)_{k \in N}$. For each $\sigma>0$, let denote by $\mu_{\sigma}$ the Gaussian probability measure on $\mathbf{R}$ with parameters $(a, \sigma)$ (here $a \in \mathbf{R}$ is fixed). Let $\left(h_{m}\right)_{m \in N}$ be a sequence of a strictly decreasing sequence of positive numbers tending to zero.

By virtue of Theorem 5.4, we know that for each $\sigma>0$ the following condition:

$$
\begin{gathered}
\mu_{\sigma}^{N}\left(\left\{\left(x_{k}\right)_{k \in N} \in \mathbf{R}^{N} \&\right.\right. \\
\left.\left.\lim _{m \rightarrow \infty} \lim _{n \rightarrow \infty} \frac{\#\left(\left\{x_{1}, \cdots, x_{n}\right\} \cap\left[a-h_{m}, a+h_{m}\right]\right)}{2 n h_{m}}=\frac{1}{\sqrt{2 \pi} \sigma}\right\}\right)=1
\end{gathered}
$$

holds true.

Let $\sigma_{0}>0$. For $\left(x_{k}\right)_{k \in N} \in \mathbf{R}^{N}$, we put

$$
T_{1}\left(\left(x_{k}\right)_{k \in N}\right)=\lim _{m \rightarrow \infty} \lim _{n \rightarrow \infty} \frac{2 n h_{m}}{\sqrt{2 \pi} \#\left(\left\{x_{1}, \cdots, x_{n}\right\} \cap\left[a-h_{m}, a+h_{m}\right]\right)},
$$

if this limit exists and belongs to the set $(0,+\infty) \backslash\left\{\sigma_{0}\right\}$, and $T_{1}\left(\left(x_{k}\right)_{k \in N}\right)=\sigma_{0}$, otherwise. Then for each $\sigma>0$, we get

$$
\mu_{\sigma}^{N}\left(\left\{\left(x_{k}\right)_{k \in N}:\left(x_{k}\right)_{k \in N} \in \mathbf{R}^{N} \& T_{1}\left(\left(x_{k}\right)_{k \in N}\right)=\sigma\right\}\right)=1,
$$

which means that $T_{1}$ is an infinite sample consistent estimate of the standard deviation $\sigma$ for the family $\left(\mu_{\sigma}^{N}\right)_{\sigma>0}$.

Theorem 5.5. Let $X_{1}, X_{2}, \cdots$ be independent normally distributed real-valued random variables with parameters $(a, \sigma)$, where a is a mean and $\sigma$ is a standard deviation. Suppose that we know the mean a. Let $\left(a_{n}\right)_{n \in N}$ be a sequence of positive numbers converging to zero and $\phi$ be a standard Gaussian density function in $\mathbf{R}$. We denote by $\mu_{\sigma}$ a Borel 
Gaussian probability measure in $\mathbf{R}$ with parameters $(a, \sigma)$ for each $\sigma \in \Sigma=(0, \infty)$. Let fix $\sigma_{0} \in \Sigma$. Let define an estimate $T_{\sigma_{0}}^{(1)}: \mathbf{R}^{N} \rightarrow \Sigma$ as follows: $T_{\sigma_{0}}^{(1)}\left(\left(x_{k}\right)_{k \in N}\right)=\varlimsup \widetilde{\lim } \widetilde{T_{n}^{(1)}}\left(\left(x_{k}\right)_{k \in N}\right)$ if $\varlimsup \widetilde{\lim } \widetilde{T_{n}^{(1)}}\left(\left(x_{k}\right)_{k \in N}\right) \in$ $\Sigma \backslash\left\{\sigma_{0}\right\} \quad$ and $T_{\sigma_{0}}^{(1)}\left(\left(x_{k}\right)_{k \in N}\right)=\sigma_{0}$, otherwise, where $\overline{\lim T_{n}^{(1)}}:=\inf _{n}$ $\sup _{m \geq n} \widetilde{T_{m}^{(1)}}$ and

$$
\widetilde{T_{n}^{(1)}}\left(\left(x_{k}\right)_{k \in N}\right)=T_{n}^{(1)}\left(x_{1}, \cdots, x_{n}\right)=\frac{1}{\sqrt{2 \pi}\left(n a_{n}\right)^{-1} \sum_{i=1}^{n} \phi\left(\frac{x_{i}-a}{a_{n}}\right)},
$$

for $\left(x_{k}\right)_{k \in N} \in \mathbf{R}^{N}$. Then $T_{\sigma_{0}}^{(1)}$ is an infinite sample consistent estimator of a parameter $\sigma$ for the family $\left(\mu_{\sigma}^{N}\right)_{\sigma \in \Sigma}$.

Proof. Following [23] (see p. 189), the function $\widetilde{\lim T T_{n}^{(1)}}$ is Borel measurable which implies that the function $\widetilde{\lim T_{n}^{(1)}}$ is $\left(\mathcal{B}\left(\mathbf{R}^{N}\right), L(\Sigma)\right)$ measurable.

For each $\sigma \in \sum$, we put

$$
A_{\sigma}=\left\{\left(x_{k}\right)_{k \in N} \in \mathbf{R}^{N}: \lim _{n \rightarrow \infty}\left(n a_{n}\right)^{-1} \sum_{i=1}^{n} \phi\left(\frac{x_{i}-a}{a_{n}}\right)=f_{\sigma}(a)\right\} .
$$

Since uniformly convergence implies pointwise convergence, by Lemma 5.1 , we deduce that $\mu_{\sigma}^{N}\left(A_{\sigma}\right)=1$ for $\sigma \in \sum$ which implies

$$
\begin{aligned}
& \mu_{\sigma}^{N}\left(\left\{\left(x_{k}\right)_{k \in N} \in \mathbf{R}^{N}: T_{\theta_{0}}^{(1)}\left(x_{k}\right)_{k \in N}=\sigma\right\}\right) \\
& \geq \mu_{\sigma}^{N}\left(\left\{\left(x_{k}\right)_{k \in N} \in \mathbf{R}^{N}: \widetilde{\lim T_{n}^{(1)}}\left(x_{k}\right)_{k \in N}=\sigma\right\}\right) \\
& \geq \mu_{\sigma}^{N}\left(\left\{\left(x_{k}\right)_{k \in N} \in \mathbf{R}^{N}: \widetilde{\lim T_{n}^{(1)}}\left(x_{k}\right)_{k \in N}=\underline{\lim } \widetilde{T_{n}^{(1)}}\left(x_{k}\right)_{k \in N}=\sigma\right\}\right)
\end{aligned}
$$




$$
\begin{aligned}
& =\mu_{\sigma}^{N}\left(\left\{\left(x_{k}\right)_{k \in N} \in \mathbf{R}^{N}: \lim _{n \rightarrow \infty} \widetilde{T_{n}^{(1)}}\left(\left(x_{k}\right)_{k \in N}\right)=\sigma\right\}\right) \\
& =\mu_{\sigma}^{N}\left(\left\{\left(x_{k}\right)_{k \in N} \in \mathbf{R}^{N}: \lim _{n \rightarrow \infty} \frac{1}{\sqrt{2 \pi}\left(n a_{n}\right)^{-1} \sum_{i=1}^{n} \phi\left(\frac{x_{i}-a}{a_{n}}\right)}=\sigma\right\}\right) \\
& =\mu_{\sigma}^{N}\left(\left\{\left(x_{k}\right)_{k \in N} \in \mathbf{R}^{N}: \lim _{n \rightarrow \infty}\left(n a_{n}\right)^{-1} \sum_{i=1}^{n} \phi\left(\frac{x_{i}-a}{a_{n}}\right)=\frac{1}{\sqrt{2 \pi} \sigma}\right\}\right) \\
& =\mu_{\sigma}^{N}\left(\left\{\left(x_{k}\right)_{k \in N} \in \mathbf{R}^{N}: \lim _{n \rightarrow \infty}\left(n a_{n}\right)^{-1} \sum_{i=1}^{n} \phi\left(\frac{x_{i}-a}{a_{n}}\right)=f_{\sigma}(a)\right\}\right) \\
& =\mu_{\sigma}^{N}\left(A_{\sigma}\right)=1 .
\end{aligned}
$$

The following theorem gives a construction of the objective infinite sample consistent estimate of an unknown parameter $\sigma$ in the same model.

Theorem 5.6. Let $X_{1}, X_{2}, \cdots$ be independent normally distributed real-valued random variables with parameters $(a, \sigma)$, where a is a mean and $\sigma$ is a standard deviation. Suppose that we know the mean a is nonzero. Let $\Phi$ be a standard Gaussian distribution function in $\mathbf{R}$. We denote by $\mu_{\sigma}$ a Borel Gaussian probability measure in $\mathbf{R}$ with parameters $(a, \sigma)$ for each $\sigma \in \Sigma=(0, \infty)$. Let fix $\sigma_{0} \in \Sigma$. Let define an estimate $T_{\sigma_{0}}^{(2)}: \mathbf{R}^{N} \rightarrow \sum$ as follows: $T_{\sigma_{0}}^{(2)}\left(\left(x_{k}\right)_{k \in N}\right)=\widetilde{\lim T_{n}^{(2)}}\left(\left(x_{k}\right)_{k \in N}\right)$ if $\widetilde{\lim } \widetilde{T_{n}^{(2)}}\left(\left(x_{k}\right)_{k \in N}\right) \in \Sigma \backslash\left\{\sigma_{0}\right\} \quad$ and $T_{\sigma_{0}}^{(2)}\left(\left(x_{k}\right)_{k \in N}\right)=\sigma_{0}$, otherwise, where $\widetilde{\lim } \widetilde{T_{n}^{(2)}}:=\inf _{n} \sup _{m \geq n} \widetilde{T_{m}^{(2)}}$ and

$$
\widetilde{T_{n}^{(2)}}\left(\left(x_{k}\right)_{k \in N}\right)=T_{n}^{(2)}\left(x_{1}, \cdots, x_{n}\right)=-\frac{a}{\Phi^{-1}\left(\frac{\#\left(\left\{x_{1}, \cdots, x_{n}\right\} \cap(-\infty, 0]\right)}{n}\right)},
$$


for $\left(x_{k}\right)_{k \in N} \in \mathbf{R}^{N}$. Then $T_{\sigma_{0}}^{(2)}$ is an objective infinite sample consistent estimator of a parameter $\sigma$ for the family $\left(\mu_{\sigma}^{N}\right)_{\sigma \in \Sigma}$.

Proof. Following [23] (see p. 189), the function $\widetilde{\lim } \widetilde{T_{n}^{(2)}}$ is Borel measurable which implies that the function $\widetilde{\lim \widetilde{T_{n}^{(2)}}}$ is $\left(\mathcal{B}\left(\mathbf{R}^{N}\right), L(\Sigma)\right)$ -measurable.

For each $\sigma \in \Sigma$, we put

$$
A_{\sigma}=\left\{\left(x_{k}\right)_{k \in N} \in \mathbf{R}^{N}:\left(x_{k}\right)_{k \in N} \text { is } \mu_{\alpha} \text {-equidistributed in } \mathbf{R}\right\} .
$$

By Corollary 2.16, we know that $\mu_{\sigma}^{N}\left(A_{\sigma}\right)=1$ for $\sigma \in \Sigma$ which implies

$$
\begin{aligned}
\mu_{\sigma}^{N} & \left(\left\{\left(x_{k}\right)_{k \in N} \in \mathbf{R}^{N}: \widetilde{\lim } \widetilde{T_{n}^{(2)}}\left(x_{k}\right)_{k \in N}=\sigma\right\}\right) \\
& \geq \mu_{\sigma}^{N}\left(\left\{\left(x_{k}\right)_{k \in N} \in \mathbf{R}^{N}: \widetilde{\lim T T_{n}^{(2)}}\left(x_{k}\right)_{k \in N}=\widetilde{\lim T T_{n}^{(2)}}\left(x_{k}\right)_{k \in N}=\sigma\right\}\right) \\
& =\mu_{\sigma}^{N}\left(\left\{\left(x_{k}\right)_{k \in N} \in \mathbf{R}^{N}: \lim _{n \rightarrow \infty} \widetilde{T_{n}^{(2)}}\left(\left(x_{k}\right)_{k \in N}\right)=\sigma\right\}\right) \\
& =\mu_{\sigma}^{N}\left(\left\{\left(x_{k}\right)_{k \in N} \in \mathbf{R}^{N}: \lim _{n \rightarrow \infty}-\frac{a}{\Phi^{-1}\left(\frac{\left(\#\left(\left\{x_{1}, \cdots x_{n}\right\} \cap(-\infty, 0]\right)\right)}{n}\right)}=\sigma\right\}\right) \\
& =\mu_{\sigma}^{N}\left(\left\{\left(x_{k}\right)_{k \in N} \in \mathbf{R}^{N}: \lim _{n \rightarrow \infty} \Phi^{-1}\left(\frac{\left(\#\left(\left\{x_{1}, \cdots x_{n}\right\} \cap(-\infty, 0]\right)\right.}{n}\right)=-\frac{a}{\sigma}\right\}\right) \\
& =\mu_{\sigma}^{N}\left(\left\{\left(x_{k}\right)_{k \in N} \in \mathbf{R}^{N}: \lim _{n \rightarrow \infty} \frac{\left(\#\left(\left\{x_{1}, \cdots x_{n}\right\} \cap(-\infty, 0]\right)\right.}{n}=\Phi\left(-\frac{a}{\sigma}\right)\right\}\right) \\
& =\mu_{\sigma}^{N}\left(\left\{\left(x_{k}\right)_{k \in N} \in \mathbf{R}^{N}: \lim _{n \rightarrow \infty} \frac{\left(\#\left(\left\{x_{1}, \cdots x_{n}\right\} \cap(-\infty, 0]\right)\right.}{n}=\Phi(a, \sigma)(0)\right\}\right) \\
& \geq \mu_{\sigma}^{N}\left(A_{\sigma}\right)=1 .
\end{aligned}
$$

The latter relation means that $\widetilde{\lim T_{n}^{(2)}}$ is a infinite-sample consistent estimate of a parameter $\sigma$ for the family of measures $\left(\mu_{\sigma}^{N}\right)_{\sigma>0}$. 
Let show that $\overline{\lim } \widetilde{T_{n}^{(2)}}$ is objective.

We have to show that for each $\sigma<0$ the set $\left(\widetilde{\lim T_{n}^{(2)}}\right)^{-1}(\sigma)$ is a Haar ambivalent set.

Let $\left(x_{k}\right)_{k \in N}$ be $\mu_{\sigma}$-equidistributed sequence. Then we get

$$
\lim _{n \rightarrow \infty} \frac{\left(\#\left(\left\{x_{1}, \cdots x_{n}\right\} \cap(-\infty, 0]\right)\right.}{n}=\Phi_{(a, \sigma)}(0),
$$

which means

$$
T_{\sigma_{0}}^{(2)}\left(\left(x_{k}\right)_{k \in N}\right)=\widetilde{\lim } \widetilde{T_{n}^{(2)}}\left(\left(x_{k}\right)_{k \in N}\right)=\sigma .
$$

Setting $J_{\sigma}=\left\{i: x_{i} \leq 0\right\}$, it is not hard to show that a set

$$
B_{J_{\sigma}}=\left\{\left(y_{i}\right)_{i \in N}: y_{i} \leq x_{i} \text { for } i \in J_{\sigma} \& y_{i}>x_{i} \text { for } i \in N \backslash J_{\sigma}\right\} \text {, }
$$

is a Haar ambivalent set.

It is clear also that for each $\left(y_{i}\right)_{i \in N} \in B_{J_{\sigma}}$, we have

$$
T_{\sigma_{0}}^{(2)}\left(\left(y_{k}\right)_{k \in N}\right)=\varlimsup \widetilde{\lim } \widetilde{T_{n}^{(2)}}\left(\left(y_{k}\right)_{k \in N}\right)=\sigma
$$

which implies that $B_{J_{\sigma}} \subseteq\left(\widetilde{\lim } \widetilde{T_{n}^{(2)}}\right)^{-1}(\sigma)$.

Since $\left\{\left(\widetilde{\lim T_{n}^{(2)}}\right)^{-1}(\sigma): \sigma>0\right\}$ is a partition of the $\mathbf{R}^{N}$ and each of them contains a Haar ambivalent set $B_{J_{\sigma}}$, we deduce that $\left(\widetilde{\lim T_{n}^{(2)}}\right)^{-1}(\sigma)$ is a Haar ambivalent set for each $\sigma>0$.

This ends the proof of the theorem.

Theorem 5.7. Let $X_{1}, X_{2}, \cdots$ be independent normally distributed real-valued random variables with parameters $(a, \sigma)$, where $a$ is a mean and $\sigma$ is a standard deviation. Suppose that both parameters are 
unknown. Let $\Phi$ be a standard Gaussian distribution function in $\mathbf{R}$. We denote by $\mu_{\sigma}$ a Borel Gaussian probability measure in $\mathbf{R}$ with parameters $(a, \sigma)$ for each $\sigma \in \Sigma=(0, \infty)$ and $a \in \mathbf{R}$. Let $\sigma_{0} \in \Sigma$. Let define an estimate $T_{\sigma_{0}}^{(3)}: \mathbf{R}^{N} \rightarrow \sum$ as follows: $T_{\sigma_{0}}^{(3)}\left(\left(x_{k}\right)_{k \in N}\right)=\widetilde{\lim T_{n}^{(3)}}\left(\left(x_{k}\right)_{k \in N}\right)$ if $\widetilde{\lim T_{n}^{(3)}}\left(\left(x_{k}\right)_{k \in N}\right) \in \sum \backslash\left\{\sigma_{0}\right\}$ and $T_{\sigma_{0}}^{(3)}\left(\left(x_{k}\right)_{k \in N}\right)=\sigma_{0}$, otherwise, where $\widetilde{\lim T_{n}^{(3)}}:=\inf _{n} \sup _{m \geq n} \widetilde{T_{m}^{(3)}}$, and

$$
\widetilde{T_{n}^{(3)}}\left(\left(x_{k}\right)_{k \in N}\right)=T_{n}^{(3)}\left(x_{1}, \cdots, x_{n}\right)=-\frac{\sum_{i=1}^{n} x_{k}}{n \Phi^{-1}\left(\frac{\#\left(\left\{x_{1}, \cdots, x_{n}\right\} \cap(-\infty, 0]\right)}{n}\right)},
$$

for $\left(x_{k}\right)_{k \in N} \in \mathbf{R}^{N}$. Then $T_{\sigma_{0}}^{(3)}$ is an infinite sample consistent estimator of a parameter $\sigma$ for the family $\left(\mu_{\sigma}^{N}\right)_{\sigma \in \Sigma}$.

Proof. Following [23] (see p. 189), the function $\widetilde{\lim T_{n}^{(3)}}$ is Borel measurable which implies that the function $\widetilde{\lim } \widetilde{T_{n}^{(3)}}$ is $\left(\mathcal{B}\left(\mathbf{R}^{N}\right), L(\Sigma)\right)$ -measurable.

For each $\sigma \in \sum$, we put

$$
A_{\sigma}=\left\{\left(x_{k}\right)_{k \in N} \in \mathbf{R}^{N}:\left(x_{k}\right)_{k \in N} \text { is } \mu_{\alpha} \text {-equidistributed in } \mathbf{R}\right\},
$$

and

$$
B_{\sigma}=\left\{\left(x_{k}\right)_{k \in N} \in \mathbf{R}^{N}: \lim _{n \rightarrow \infty} \frac{\sum_{k=1}^{n} x_{k}}{n}=a\right\} .
$$

On the one hand, by By Corollary 2.16, we know that $\mu_{\sigma}^{N}\left(A_{\sigma}\right)=1$ for $\sigma \in \sum$. On the other hand, by strong law of large numbers, we know that $\mu_{\sigma}^{N}\left(B_{\sigma}\right)=1$ for $\sigma \in \sum$. These relations imply that 


$$
\mu_{\sigma}^{N}\left(A_{\sigma} \cap B_{\sigma}\right)=1,
$$

for $\sigma \in \sum$.

Take into account (5.22), we get

$$
\begin{aligned}
\mu_{\sigma}^{N} & \left(\left\{\left(x_{k}\right)_{k \in N} \in \mathbf{R}^{N}: \widetilde{\lim } \widetilde{T_{n}^{(3)}}\left(x_{k}\right)_{k \in N}=\sigma\right\}\right) \\
& \geq \mu_{\sigma}^{N}\left(\left\{\left(x_{k}\right)_{k \in N} \in \mathbf{R}^{N}: \widetilde{\lim T_{n}^{(3)}}\left(x_{k}\right)_{k \in N}=\underline{\lim T T_{n}^{(3)}}\left(x_{k}\right)_{k \in N}=\sigma\right\}\right) \\
& =\mu_{\sigma}^{N}\left(\left\{\left(x_{k}\right)_{k \in N} \in \mathbf{R}^{N}: \lim _{n \rightarrow \infty} \widetilde{T_{n}^{(3)}}\left(\left(x_{k}\right)_{k \in N}=\sigma\right\}\right)\right. \\
& =\mu_{\sigma}^{N}\left(\left\{\left(x_{k}\right)_{k \in N} \in \mathbf{R}^{N}: \lim _{n \rightarrow \infty}-\frac{\sum_{k=1}^{n} x_{k}}{n} \Phi^{-1}\left(\frac{\left(\#\left(\left\{x_{1}, \cdots, x_{n}\right\} \cap(-\infty, 0]\right)\right)}{n}=\sigma\right\}\right)\right. \\
& \geq \mu_{\sigma}^{N}\left(\left\{\left(x_{k}\right)_{k \in N} \in A_{\sigma} \cap B_{\sigma}: \lim _{n \rightarrow \infty} \Phi^{-1}\left(\frac{\left(\#\left(\left\{x_{1}, \cdots, x_{n}\right\} \cap(-\infty, 0]\right)\right.}{n}\right)\right.\right. \\
& \left.\left.=-\lim _{n \rightarrow \infty} \frac{\sum_{k=1}^{n} x_{k}}{\sigma}\right\}\right) \\
& =\mu_{\sigma}^{N}\left(\left\{\left(x_{k}\right)_{k \in N} \in A_{\sigma} \cap B_{\sigma}: \lim _{n \rightarrow \infty} \Phi^{-1}\left(\frac{\left(\#\left(\left\{x_{1}, \cdots, x_{n}\right\} \cap(-\infty, 0]\right)\right.}{n}\right)=-\frac{a}{\sigma}\right\}\right) \\
& =\mu_{\sigma}^{N}\left(\left\{\left(x_{k}\right)_{k \in N} \in \mathbf{R}^{N}: \lim _{n \rightarrow \infty} \frac{\left(\#\left(\left\{x_{1}, \cdots, x_{n}\right\} \cap(-\infty, 0]\right)\right.}{n}=\Phi_{(a, \sigma)}(0)\right\}\right) \\
& =\mu_{\sigma}^{N}\left(A_{\sigma} \cap B_{\sigma}\right)=1 .
\end{aligned}
$$

The latter relation means that $T_{\sigma_{0}}^{(3)}$ is an infinite-sample consistent estimate of a parameter $\sigma$ for the family of measures $\left(\mu_{\sigma}^{N}\right)_{\sigma>0}$.

This ends the proof of the theorem. 
Example 5.8. Since a sequence of real numbers $(\pi \times n-[\pi \times n])_{n \in N}$, where [.] denotes an integer part of a real number, is uniformly distributed on $(0,1)$ (see [10], Example 2.1, p. 17), we claim that a simulation of a $\mu_{(3,5)}$-equidistributed sequence $\left(x_{n}\right)_{n \leq M}$ on $R$ ( $M$ is a "sufficiently large" natural number and depends on a representation quality of the irrational number $\pi$ ), where $\mu_{(3,5)}$ denotes a a linear Gaussian measure with parameters $(3,5)$, can be obtained by the formula

$$
x_{n}=\Phi_{(3,5)}^{-1}(\pi \times n-[\pi \times n]),
$$

for $n \leq M$, where $\Phi_{(3,5)}$ denotes a Gaussian distribution function with parameters $(3,5)$.

Suppose that we know a mean $a=3$ and want to estimate an "unknown" standard deviation $\sigma$.

We set: $n$ - the number of trials; $S_{n}$ - a square root from the sample variance; $S_{n}^{\prime}$ - a square root from the corrected sample variance; $T_{n}^{(2)}$ an estimate defined by the formula (5.13); $T_{n}^{(3)}$ - an estimate defined by the formula (5.19); and $\sigma$ - an unknown standard deviation.

The numerical data placed in Table 3 were obtained by using Microsoft Excel. Notice that results of computations presented in Table 3 show us that both statistics $T_{n}^{(2)}$ and $T_{n}^{(3)}$ work correctly. Unfortunately, we can not present the results of computations for the statistics $T_{n}^{(1)}$ defined by the formula (5.10) because it needs a quite exact calculations. 
Table 3. Estimates of an unknown standard deviation $\sigma=5$

\begin{tabular}{ccccc}
\hline $\boldsymbol{n}$ & $S_{n}$ & $S_{n}^{\prime}$ & $T_{n}^{(2)}$ & $T_{n}^{(3)}$ \\
\hline 200 & 4.992413159 & 5.004941192 & 5.205401325 & 4.895457577 \\
400 & 4.992413159 & 5.004941192 & 5.141812921 & 4.835655399 \\
600 & 5.10523925 & 5.109498942 & 5.211046737 & 4.855457413 \\
800 & 5.106390271 & 5.109584761 & 5.19369988 & 4.92581015 \\
1000 & 5.066642282 & 5.069177505 & 5.028142523 & 4.944169095 \\
1200 & 5.072294934 & 5.074409712 & 5.235885276 & 4.935995814 \\
1400 & 5.081110418 & 5.082926073 & 5.249446371 & 4.96528786 \\
1600 & 5.079219075 & 5.080807075 & 5.205452797 & 4.9564705 \\
1800 & 5.060850283 & 5.06225666 & 5.207913228 & 4.963326232 \\
2000 & 5.063112113 & 5.064378366 & 5.239119585 & 4.981223889 \\
\hline
\end{tabular}

At end of this section, we state the following:

Problem 5.5. Let $\mathcal{D}$ be a class of positive continuous probability densities and $p_{f}$ be a Borel probability measure on $\mathbf{R}$ with probability density function $f$ for each $f \in \mathcal{D}$. Does there exist an objective (or a subjective) infinite sample consistent estimate of an unknown probability density function $f$ for the family of Borel probability measures $\left\{p_{f}^{N}: f \in \mathcal{D}\right\} ?$

\section{On Orthogonal Statistical Structures in a Non-Locally- Compact Polish Group Admitting an Invariant Metric}

Let $G$ be a Polish group, by which we mean a separable group with a complete invariant metric $\rho$ (i.e., $\rho\left(f h_{1} g, f h_{2} g\right)=\rho\left(h_{1}, h_{2}\right)$ for each $f, g, h_{1}, h_{2} \in G$ ) for which the transformation (from $G \times G$ onto $G$ ), which sends $(x, y)$ into $x^{-1} y$ is continuous. Let $\mathcal{B}(G)$ denotes the $\sigma$-algebra of Borel subsets of $G$. 
Definition 6.1 ([12]). A Borel set $X \subseteq G$ is called shy, if there exists a Borel probability measure $\mu$ over $G$ such that $\mu(f X g)=0$ for all $f, g \in G$. A measure $\mu$ is called a testing measure for a set $X$. A subset of a Borel shy set is called also shy. The complement of a shy set is called a prevalent set.

Definition 6.2 ([1]). A Borel set is called a Haar ambivalent set if it is neither shy nor prevalent.

Remark 6.3. Notice that if $X \subseteq G$ is shy then there exists such a testing measure $\mu$ for a set $X$ which has a compact carrier $K \subseteq G$ (i.e., $\mu(G \backslash K)=0)$. The collection of shy sets constitutes an $\sigma$-ideal and in the case where $G$ is locally compact a set is shy iff it has Haar measure zero.

Definition 6.4. If $G$ is a Polish group and $\left\{\mu_{\theta}: \theta \in \Theta\right\}$ is a family of Borel probability measures on $G$, then the family of triplets $\left\{\left(G, \mathcal{B}, \mu_{\theta}\right)\right.$ : $\theta \in \Theta\}$, where $\Theta$ is a non-empty set equipped with an $\sigma$ algebra $L(\Theta)$ generated by all singletons of $\Theta$, is called a statistical structure. A set $\Theta$ is called a set of parameters.

Definition 6.5. $(\mathcal{O})$ The statistical structure $\left\{\left(G, \mathcal{B}(G), \mu_{\theta}\right): \theta \in \Theta\right\}$ is called orthogonal if the measures $\mu_{\theta_{1}}$ and $\mu_{\theta_{2}}$ are orthogonal for each different parameters $\theta_{1}$ and $\theta_{2}$.

Definition 6.6. $(\mathcal{W S})$ The statistical structure $\left\{\left(G, \mathcal{B}(G), \mu_{\theta}\right): \theta \in \Theta\right\}$ is called weakly separated if there exists a family of Borel subsets $\left\{X_{\theta}: \theta \in \Theta\right\} \quad$ such that $\mu_{\theta_{1}}\left(X_{\theta_{2}}\right)=\delta\left(\theta_{1}, \theta_{2}\right)$, where $\delta$ denotes Kroneckers function defined on the Cartesian square $\Theta \times \Theta$ of the set $\Theta$.

Definition 6.7. $(\mathcal{S S})$ The statistical structure $\left\{\left(G, \mathcal{B}(G), \mu_{\theta}\right): \theta \in \Theta\right\}$ is called strong separated (or strictly separated) if there exists a partition of the group $G$ into family of Borel subsets $\left\{X_{\theta}: \theta \in \Theta\right\}$ such that $\mu_{\theta}\left(X_{\theta}\right)=1$ for each $\theta \in \Theta$. 
Definition 6.8. $(\mathcal{C E})$ A $(\mathcal{B}(G), L(\Theta))$-measurable mapping $T: G \rightarrow \Theta$ is called a consistent estimate of an unknown parameter $\theta \in \Theta$ for the statistical structure $\left\{\left(G, \mathcal{B}(G), \mu_{\theta}\right): \theta \in \Theta\right\}$ if the condition $\mu_{\theta}\left(T^{-1}(\theta)\right)=1$ holds true for each $\theta \in \Theta$.

Definition 6.9. $(\mathcal{O C E})$ A $(\mathcal{B}(G), L(\Theta))$-measurable mapping $T: G \rightarrow \Theta$ is called an objective consistent estimate of an unknown parameter $\theta \in \Theta$ for the statistical structure $\left\{\left(G, \mathcal{B}(G), \mu_{\theta}\right): \theta \in \Theta\right\}$ if the following two conditions hold:

(i) $\mu_{\theta}\left(T^{-1}(\theta)\right)=1$ for each $\theta \in \Theta$;

(ii) $T^{-1}(\theta)$ is a Haar ambivalent set for each $\theta \in \Theta$.

If the condition (i) holds but the condition (ii) fails, then $T$ is called a subjective consistent estimate of an unknown parameter $\theta \in \Theta$ for the statistical structure $\left\{\left(G, \mathcal{B}, \mu_{\theta}\right): \theta \in \Theta\right\}$.

Definition 6.10. $(\mathcal{S O C E})$ An objective consistent estimate $T: G \rightarrow \Theta$ of an unknown parameter $\theta \in \Theta$ for the statistical structure $\{(G, \mathcal{B}(G)$, $\left.\left.\mu_{\theta}\right): \theta \in \Theta\right\}$ is called strong if for each $\theta_{1}, \theta_{2} \in \Theta$ there exists an isometric Borel measurable bijection $A_{\left(\theta_{1}, \theta_{2}\right)}: G \rightarrow G$ such that the set $A_{\left(\theta_{1}, \theta_{2}\right)}\left(T^{-1}(\theta)\right) \Delta T^{-1}\left(\theta_{2}\right)$ is shy in $G$.

Remark 6.11. Let $G$ be a Polish non-locally-compact group admitting an invariant metric. The relations between statistical structures introduced in Definitions 6.5-6.10 for such a group can be presented by the following diagram:

$$
\mathcal{S O C E} \rightarrow \mathcal{O C E} \rightarrow \mathcal{C E} \leftrightarrow \mathcal{S S} \rightarrow \mathcal{W S} \rightarrow \mathcal{O} .
$$

To show that the converse implications sometimes fail we consider the following examples: 
Example 6.12. $\rceil(\mathcal{W S} \leftarrow \mathcal{O})$ Let $F \subset G$ be a closed subset of the cardinality $2^{\aleph_{0}}$. Let $\phi:[0,1] \rightarrow F$ be a Borel isomorphism of $[0,1]$ onto $F$. We set $\mu(X)=\lambda\left(\phi^{-1}(X \cap F)\right)$ for $X \in \mathcal{B}(G)$, where $\lambda$ denotes a linear Lebesgue measure on $[0,1]$. We put $\Theta=F$. Let $\theta_{0} \in \Theta$ and put: $\mu_{\theta}=\mu$ if $\theta=\theta_{0}$, and $\mu_{\theta}=\left.\delta_{\theta}\right|_{\mathcal{B}(G)}$, otherwise, where $\delta_{\theta}$ denotes a Dirac measure on $G$ concentrated at the point $\theta$ and $\left.\delta_{\theta}\right|_{\mathcal{B}(G)}$ denotes the restriction of the $\delta_{\theta}$ to the class $\mathcal{B}(G)$. Then the statistical structure $\left\{\left(G, \mathcal{B}, \mu_{\theta}\right): \theta \in \Theta\right\}$ stands $\mathcal{O}$ which is not $\mathcal{W S}$.

Example 6.13. (SM) $\urcorner(\mathcal{S S} \leftarrow \mathcal{W S})$ Following [15] (see Theorem 1, p. 335), in the system of axioms $(Z F C)$ the following three conditions are equivalent:

(1) The continuum hypothesis $\left(c=2^{\aleph_{0}}=\aleph_{1}\right)$.

(2) For an arbitrary probability space $(E ; S ; \mu)$, the $\mu$-measure of the union of any family $\left(E_{i}\right)_{i \in I}$ of $\mu$-measure zero subsets, such that $\operatorname{card}(I)<c$, is equal to zero.

(3) an arbitrary weakly separated family of probability measures, of cardinality continuum, is strictly separated.

The latter relation means that under continuum hypothesis in ZFC we have $\mathcal{S S} \leftarrow \mathcal{W S}$. This is just Skorohod well known result (see [26]). Moreover, following [15] (see Theorem 2, p.339), if $(F, \rho)$ is a Radon metric space and $\left(\mu_{i}\right)_{i \in I}$ is a weakly separated family of Borel probability measures with $\operatorname{card}(I) \leq c$, then in the system of axioms $(Z F C) \&(M A)$, the family $\left(\mu_{i}\right)_{i \in I}$ is strictly separated.

Let consider a counter example to the implication $\mathcal{S S} \leftarrow \mathcal{W S}$ in the Solovay model $(S M)$ [25], which is the following system of axioms: $(Z F)+$ $D C+$ "every subset of the real axis $\mathbf{R}$ is Lebesgue measurable", where $(Z F)$ denotes the Zermelo-Fraenkel set theory and $(D C)$ denotes the axiom of dependent choices. 
For $\theta \in(0 ; 1)$, let $b_{\theta}$ be a linear classical Borel measure defined on the set $\{\theta\} \times(0 ; 1)$. For $\theta \in(1 ; 2)$, let $b_{\theta}$ be a linear classical Borel measure defined on the set $(0 ; 1) \times\{\theta-1\}$. By $\lambda_{\theta}$ we denote a Borel probability measure on $(0 ; 1) \times(0 ; 1)$ produced by $b_{\theta}$, i.e.,

$$
\begin{aligned}
& (\forall X)\left(\forall \theta_{1}\right)\left(\forall \theta_{2}\right)\left(X \in \mathcal{B}((0 ; 1) \times(0 ; 1)) \& \theta_{1} \in(0 ; 1) \& \theta_{2} \in(1 ; 2) \rightarrow\right. \\
& \left.\lambda_{\theta_{1}}(X)=b_{\theta_{1}}\left(\left(\left\{\theta_{1}\right\} \times(0 ; 1)\right) \cap X\right) \& \lambda_{\theta_{2}}(X)=b_{\theta_{2}}\left(\left((0 ; 1) \times\left\{\theta_{1}-1\right\}\right) \cap X\right)\right) .
\end{aligned}
$$

If we put $\theta=(0 ; 1) \cup(1 ; 2)$, then we get a statistical structure

$$
\left((0 ; 1) \times(0 ; 1), \mathcal{B}((0 ; 1) \times(0 ; 1)), \lambda_{\theta}\right)_{\theta \in \Theta} .
$$

Setting $X_{\theta}=\{\theta\} \times(0 ; 1)$ for $\theta \in(0 ; 1)$, and $X_{\theta}=(0 ; 1) \times\{\theta-1\}$ for $\theta \in(1.2)$, we observe that for the family of Borel subsets $\left\{X_{\theta}: \theta \in \Theta\right\}$ we have $\lambda_{\theta_{1}}\left(X_{\theta_{2}}\right)=\delta\left(\theta_{1}, \theta_{2}\right)$, where $\delta$ denotes Kroneckers function defined on the Cartesian square $\Theta \times \Theta$ of the set $\Theta$. In the other words, $\left(\lambda_{\theta}\right)_{\theta \in \Theta}$ is weakly separated. Now, let assume that this family is strong separated. Then, there will be a partition $\left\{Y_{\theta}: \theta \in \Theta\right\}$ of the set $(0 ; 1) \times$ $(0 ; 1)$ into Borel subsets $\left(Y_{\theta}\right)_{\theta \in \Theta}$ such that $\lambda_{\theta}\left(Y_{\theta}\right)=1$ for each $\theta \in \Theta$. If we consider $A=\bigcup_{\theta \in(0 ; 1)} Y_{\theta}$ and $B=\bigcup_{\theta \in(1 ; 2)} Y_{\theta}$, then we observe by Fubini theorem that $\ell_{2}(A)=1$ and $\ell_{2}(B)=1$, where $\ell_{2}$ denotes the 2 -dimensional Lebesgue measure defined in $(0 ; 1) \times(0 ; 1)$. This is the contradiction and we proved that $\left(\lambda_{\theta}\right)_{\theta \in \Theta}$ is not strictly separated. An existence of a Borel isomorphism $g$ between $(0 ; 1) \times(0 ; 1)$ and $G$ allows us to construct a family $\left(\mu_{\theta}\right)_{\theta \in \Theta}$ in $G$ as follows: $\mu_{\theta}(X)=\lambda_{\theta}\left(g^{-1}(X)\right)$ for each $X \in \mathcal{B}(G)$ and $\theta \in \Theta$, which is $\mathcal{W S}$ but no $\mathcal{S S}$ (equivalently, $\mathcal{C}$ ). By virtue the celebrated result of Mycielski and Swierczkowski (see [11]) asserted that under the axiom of determinacy $(A D)$ every subset of the real axis $\mathbf{R}$ is Lebesgue measurable, the same example can be used as a 
counter example to the implication $\mathcal{S S} \leftarrow \mathcal{W S}$ in the theory (ZF) + (DC) $+(\mathrm{AD})$. Since the answer to the question asking "whether $\left(\mu_{\theta}\right)_{\theta \in \Theta}$ has a consistent estimate?" is yes in the theory $(\mathrm{ZFC}) \&(\mathrm{CH})$, and no in the theory $(Z F)+(D C)+(A D)$, we deduce that this question is not solvable within the theory $(Z F)+(D C)$.

Example 6.14. $\rceil(\mathcal{O C E} \leftarrow \mathcal{C E})$ Setting $\Theta=G$ and $\mu_{\theta}=\delta_{\theta} \mid \mathcal{B}(G)$ for $\theta \in \Theta$, where $\delta_{\theta}$ denotes a Dirac measure in $G$ concentrated at the point $\theta$ and $\delta_{\theta} \mid \mathcal{B}(G)$ denotes its restriction to $\mathcal{B}(G)$, we get a statistical structure $\left(G, \mathcal{B}(G), \mu_{\theta}\right)_{\theta \in \Theta}$. Let $L(\Theta)$ denotes a minimal $\sigma$-algebra of subsets of $\Theta$ generated by all singletons of $\Theta$. Setting $T(g)=g$ for $g \in G$, we get a consistent estimate of an unknown parameter $\theta$ for the family $\left(\mu_{\theta}\right)_{\theta \in \Theta}$. Notice that there does not exist an objective consistent estimate of a parameter $\theta$ for the family $\left(\mu_{\theta}\right)_{\theta \in \Theta}$. Indeed, if we assume the contrary and $T_{1}$ be such an estimate, we get that $T_{1}^{-1}(\theta)$ is a Haar ambivalent set for each $\theta \in \Theta$. Since $T_{1}$ is a consistent estimate of an unknown parameter $\theta$ for each $\theta \in \Theta$, we get that the condition $\mu_{\theta}\left(T_{1}^{-1}(\theta)\right)=1$ holds true which implies that $\theta \in T_{1}^{-1}(\theta)$ for each $\theta \in \Theta$. Let fix any parameter $\theta_{0} \in \Theta$. Since $T_{1}^{-1}\left(\theta_{0}\right)$ is a Haar ambivalent set there is $\theta_{1} \in T_{1}^{-1}\left(\theta_{0}\right)$ which differs from $\theta_{0}$. Then $T_{1}^{-1}\left(\theta_{0}\right)$ and $T_{1}^{-1}\left(\theta_{1}\right)$ are not disjoint because $\theta_{1} T_{1}^{-1}\left(\theta_{0}\right) \cap T_{1}^{-1}\left(\theta_{1}\right)$ and we get the contradiction.

Remark 6.15. Notice that if $(\Theta, \rho)$ is a metric space and if in the Definition 2.8 the requirement of a $(\mathcal{B}(G), L(\Theta))_{i}$-measurability will be replaced with a $(\mathcal{B}(G), \mathcal{B}(\Theta))$-measurability, then the implication $\mathcal{S S} \rightarrow \mathcal{C E}$ may be false. Indeed, let $G$ be a Polish group and $f: G \leftarrow \Theta(:=G)$ be a non-measurable (in the Borel sense) bijection. For each $\theta \in \Theta$ denote by $\mu_{\theta}$ the restriction of the Dirac measure $\delta_{f(\theta)}$ to the $\sigma$-algebra of Borel subsets of the group $G$. It is clear that the statistical structure 
$\left\{\left(G, \mathcal{B}(G), \mu_{\theta}\right): \theta \in \Theta\right\}$ is strictly separated. Let show that there does not exist a consistent estimate for that statistical structure. Indeed, let $T: G \rightarrow \Theta$ be $(\mathcal{B}(G), \mathcal{B}(\Theta))$-measurable mapping such that $\mu_{\theta}(\{x: T$ $(x)=\theta\})=1$ for each $\theta \in \Theta$. Since the measure $\mu_{\theta}$ is concentrated at the point $f(\theta)$, we have that $f(\theta) \in\{x: T(x)=\theta\}$ for each $\theta \in \Theta$ which implies that $T(f(\theta))=\theta$ for each $\theta \in \Theta$. The latter relation means that $T=f^{-1}$. Since $f$ is not $(\mathcal{B}(G), \mathcal{B}(\Theta))$-measurable we claim that $f^{-1}=T$ is not also $(\mathcal{B}(G), \mathcal{B}(\Theta))$-measurable and we get the contradiction.

There naturally arises a question asking whether there exists such a statistical structure $\left\{\left(G, \mathcal{B}, \mu_{\theta}\right): \theta \in \Theta\right\}$ in a Polish non-locally-compact group admitting an invariant metric, which has an objective consistent estimate of a parameter $\theta$. To answer positively to this question, we need the following two lemmas:

Lemma 6.16 ([24], Theorem, p. 206). Assume $G$ is a Polish nonlocally-compact group admitting an invariant metric. Then, there exists a closed set $F \subseteq G$ and a continuous function $\phi: F \rightarrow 2^{N}$ such that for any $x \in 2^{N}$ and any compact set $K \subseteq G$, there is $g \in G$ with $g K \subseteq \phi^{-1}(x)$.

Lemma 6.17 ([5], Proposition 12, p.87). Let $G$ be a non-locallycompact Polish group with an invariant metric. Then any compact subset (and hence any $K_{\sigma}$ subset) of $G$ is shy.

Remark 6.18. In [19] (see proof of Theorem 4.1, Step 2) has been constructed a partition $\Phi=\left\{A_{\theta}: \theta \in[0,1]\right\}$ of the $\mathbf{R}^{N}$ into Haar ambivalent sets such that for each $\theta_{1}, \theta_{2} \in[0,1]$, there exists an isometric (with respect to Tychonoff metric which is invariant under translates) Borel measurable bijection $A_{\left(\theta_{1}, \theta_{2}\right)}$ of $\mathbf{R}^{N}$ such that $A_{\left(\theta_{1}, \theta_{2}\right)}\left(A_{\theta_{1}}\right) \Delta A_{\theta_{2}}$ is shy. In this context and concerning with Lemma 
6.16, it is natural to ask whether an arbitrary Polish non-locally-compact group with an invariant metric admits a similar partition into Haar ambivalent sets. Notice that we have no any information in this direction.

Theorem 6.19. Let $G$ be a Polish non-locally-compact group admitting an invariant metric. Then, there exists a statistical structure $\left\{\left(G, \mathcal{B}, \mu_{\theta}\right): \theta \in \Theta\right\}$ in $G$ which has an objective consistent estimate of a parameter $\theta$ such that:

(i) $\Theta \subseteq G$ and $\operatorname{card}(\Theta)=2^{\aleph_{0}}$;

(ii) $\mu_{\theta}$ is the restriction of the Dirac measure concentrated at the point $\theta$ to the Borel $\sigma$-algebra $\mathcal{B}(G)$ for each $\theta \in \Theta$.

Proof. By virtue of Lemma 6.16, there exists a closed set $F \subseteq G$ and a continuous function $\phi: F \rightarrow 2^{N}$ such that for any $x \in 2^{N}$ and any compact set $K \subseteq G$, there is $g \in G$ with $g K \subseteq \phi^{-1}(x)$. For $x \in 2^{N} \backslash\{(0,0, \ldots)\}$, we put

$$
X_{x}=\phi^{-1}(x)
$$

We set $X_{(0,0, \ldots)}=\phi^{-1}((0,0, \cdots)) \cup(G \backslash F)$. Thus, we have a partition $\left\{X_{x}: x \in 2^{N}\right\}$ of $G$ into Borel subsets such that each element of the partition is Borel measurable and Haar ambivalent set. Let $\left\{\theta_{x}: x \in 2^{N}\right\}$ be any selector. We put $\Theta=\left\{\theta: \theta=\theta_{x}\right.$ for some $\left.x \in 2^{N}\right\}$ and denote by $\mu_{\theta}$ the restriction of the Dirac measure concentrated at the point $\theta$ to the $\sigma$-algebra $\mathcal{B}(G)$. Thus, we have constructed a statistical structure $\left\{\left(G, \mathcal{B}, \mu_{\theta}\right): \theta \in \Theta\right\}$ in $G$. We put $T(g)=\theta$ for each $g \in X_{\theta}$. Now, it is obvious that $T$ is the objective consistent estimate of a parameter $\theta$ for the statistical structure $\left\{\left(G, \mathcal{B}, \mu_{\theta}\right): \theta \in \Theta\right\}$ in $G$ such that the conditions (i)-(ii) are fulfilled. 
Theorem 6.20. Let $G$ be a Polish non-locally-compact group admitting an invariant metric. Let $\mu$ be a Borel probability measure whose carrier is a compact set $K_{0}\left(\right.$ i.e., $\left.\mu\left(G \backslash K_{0}\right)=0\right)$. Then, there exists a statistical structure $\left\{\left(G, \mathcal{B}, \mu_{\theta}\right): \theta \in \Theta\right\}$ in $G$ which has an objective consistent estimate of a parameter $\theta$ such that

(i) $\Theta \subseteq G$ and $\operatorname{card}(\Theta)=2^{\aleph_{0}}$;

(ii) $\mu_{\theta}$ is a $\theta$-shift of the measure $\mu\left(\right.$ i.e., $\mu_{\theta}(X)=\mu\left(\theta^{-1} X\right)$ for $X \in \mathcal{B}(G)$ and $\theta \in \Theta)$.

Proof. By virtue of Lemma 6.16, there exists a closed set $F \subseteq G$ and a continuous function $\phi: F \rightarrow 2^{N}$ such that for any $x \in 2^{N}$ and any compact set $K \subseteq G$, there is $g \in G$ with $g K \subseteq \phi^{-1}(x)$. For $x \in 2^{N}$ $\backslash\{(0,0, \cdots)\}$, we put $X_{x}=\phi^{-1}(x)$. We set $X_{(0,0, \ldots)}=\phi^{-1}((0,0, \cdots)) \cup$ $(G \backslash F)$. Thus, we have a partition $\left\{X_{x}: x \in 2^{N}\right\}$ of $G$ into Borel subsets such that each element of the partition is Borel measurable, Haar ambivalent set and for any $x \in 2^{N}$ and any compact set $K \subseteq G$, there is $g \in G$ with $g K \subseteq X_{x}$. If we take under $K$ a set $K_{0}$, then for any $x \in 2^{N}$, there is $g\left(K_{0}, x\right) \in G$ with $g\left(K_{0}, x\right) K_{0} \subseteq X_{x}$. We put $\Theta=\left\{\theta: \theta=g\left(K_{0}, x\right) \& x \in 2^{N}\right\}$. For each $\theta \in \Theta$ and $X \in \mathcal{B}(G)$, we put $\mu_{\theta}(X)=\mu\left(\theta^{-1} X\right)$. For $g \in X_{x}$, we put $T(g)=g\left(K_{0}, x\right)$. Let us show that $T: G \rightarrow \Theta$ is an objective consistent estimate of a parameter $\theta$. Indeed, on the one hand, for each $\theta \in \Theta$, we have

$$
\begin{aligned}
\mu_{\theta}\left(T^{-1}(\theta)\right) & =\mu_{g\left(K_{0}, x\right)}\left(T^{-1}\left(g\left(K_{0}, x\right)\right)\right)=\mu_{g\left(K_{0}, x\right)}\left(X_{x}\right)=\mu\left(g\left(K_{0}, x\right)^{-1} X_{x}\right) \\
& \geq \mu\left(g\left(K_{0}, x\right)^{-1} g\left(K_{0}, x\right) K_{0}\right)=\mu\left(K_{0}\right)=1
\end{aligned}
$$

which means that $T: G \rightarrow \Theta$ is a consistent estimate of a parameter $\theta$. On the other hand, for each $\theta=g\left(K_{0}, x\right) \in \Theta$, we have that a set $T^{-1}(\theta)$ 
$=T^{-1}\left(g\left(K_{0}, x\right)\right)=X_{x}$ is Borel measurable and a Haar ambivalent set which together with the latter relation implies that $T: G \rightarrow \Theta$ is an objective consistent estimate of a parameter $\theta$. Now, it is obvious to check that for the statistical structure $\left\{\left(G, \mathcal{B}, \mu_{\theta}\right): \theta \in \Theta\right\}$ the conditions (i)-(ii) are fulfilled.

The next theorem shows whether can be constructed an objective consistent estimates by virtue of some consistent estimates in a Polish non-locally-compact group admitting an invariant metric.

Theorem 6.21. Assume $G$ is a Polish non-locally-compact group admitting an invariant metric. Let $\operatorname{card}(\Theta)=2^{\aleph_{0}}$ and $T: G \rightarrow \Theta$ be a consistent estimate of a parameter $\theta$ for the family of Borel probability measures $\left(\mu_{\theta}\right)_{\theta \in \Theta}$ such that there exists $\theta_{0} \in \Theta$ for which $T^{-1}\left(\theta_{0}\right)$ is a prevalent set. Then, there exists an objective consistent estimate of a parameter $\theta$ for the family $\left(\mu_{\theta}\right)_{\theta \in \Theta}$.

Proof. For $\theta \in \Theta$ we put $S_{\theta}=T^{-1}(\theta)$. Since $S_{\theta_{0}}$ is a prevalent set, we deduce that

$$
\bigcup_{\theta \in \Theta \backslash\left\{\theta_{0}\right\}} S_{\theta}=\mathbf{R}^{N} \backslash S_{\theta_{0}}
$$

is shy in $G$.

By Lemma 2.6, we know that the measure $\mu_{\theta_{0}}$ is concentrated on a union of a countable family of compact subsets $\left\{F_{k}^{\left(\theta_{0}\right)}: k \in N\right\}$. By Lemma 6.7, we know that $\bigcup_{k \in N} F_{k}^{\left(\theta_{0}\right)}$ is shy in $G$.

We put $\widetilde{S}_{\theta}=S_{\theta}$ for $\theta \in \Theta \backslash\left\{\theta_{0}\right\}$ and $\widetilde{S}_{\theta_{0}}=\bigcup_{k \in N} F_{k}^{\left(\theta_{0}\right)}$. Clearly, $S=\bigcup_{\theta \in \Theta} \widetilde{S}_{\theta}$ is shy in $G$.

By virtue of Lemma 6.16, there exists a closed set $F \subseteq G$ and a continuous function $\phi: F \rightarrow 2^{N}$ such that for any $x \in 2^{N}$ and any 
compact set $K \subseteq G$, there is $g \in G$ with $g K \subseteq \phi^{-1}(x)$. Let $f: 2^{N} \rightarrow \Theta$ be any bijection. For $\theta \in \Theta$, we put

$$
B_{\theta}=\left(\phi^{-1}\left(f^{-1}(\theta)\right) \backslash S\right) \cup S_{\theta}
$$

Notice that $\left(B_{\theta}\right)_{\theta \in \Theta}$ is a partition of $G$ into Haar ambivalent sets. We put $T_{1}(g)=\theta$ for $g \in B_{\theta}(\theta \in \Theta)$. Since

$$
\mu_{\theta}\left(T_{1}^{-1}(\theta)\right)=\mu_{\theta}\left(B_{\theta}\right) \geq \mu_{\theta}\left(S_{\theta}\right)=1,
$$

for $\theta \in \Theta$, we claim that $T_{1}$ is a consistent estimate of a parameter $\theta$ for the family $\left(\mu_{\theta}\right)_{\theta \in \Theta}$. Since $T_{1}^{-1}(\theta)=B_{\theta}$ is a Borel and Haar ambivalent set for each $\theta \in \Theta$ we end the proof of the theorem.

Example 6.22. Let $F$ be a distribution function on $\mathbf{R}$ such that the integral $\int_{\mathbf{R}} x d F(x)$ exists and is equal to zero. Suppose that $p$ is a Borel probability measure on $\mathbf{R}$ defined by $F$. For $\theta \in \Theta(:=\mathbf{R})$, let $p_{\theta}$ be $\theta$-shift of the measure $p\left(\right.$ i.e., $p_{\theta}(X)=p(X-\theta)$ for $\left.X \in \mathcal{B}(\mathbf{R})\right)$. Setting, $G=\mathbf{R}^{N}$, for $\theta \in \Theta$, we put $\mu_{\theta}=p_{\theta}^{N}$, where $p_{\theta}^{N}$ denotes the infinite power of the measure $p_{\theta}$. We set $T\left(\left(x_{k}\right)_{k \in N}\right)=\lim _{n \rightarrow \infty} \frac{\sum_{k=1}^{n} x_{k}}{n}$, if $\lim _{n \rightarrow \infty} \frac{\sum_{k=1}^{n} x_{k}}{n}$ exists, is finite and differs from the zero, and $T\left(\left(x_{k}\right)_{k \in N}\right)=0$, otherwise. Notice that $T: \mathbf{R}^{N} \rightarrow \Theta$ is a consistent estimate of a parameter $\theta$ for the family $\left(\mu_{\theta}\right)_{\theta \in \Theta}$ such that $T^{-1}(0)$ is a prevalent set. Indeed, by virtue the strong law of large numbers, we know that

$$
\mu_{\theta}\left(\left\{\left(x_{k}\right)_{k \in N}: \lim _{n \rightarrow \infty} \frac{\sum_{k=1}^{n} x_{k}}{n}=\theta\right\}\right)=1,
$$

for $\theta \in \Theta$. 
Following [18] (Lemma 4.14, p. 60), a set $S$ defined by

$$
S=\left\{\left(x_{k}\right)_{k \in N}: \lim _{n \rightarrow \infty} \frac{\sum_{k=1}^{n} x_{k}}{n} \text { exists and is finite }\right\},
$$

is Borel shy set, which implies that $\mathbf{R}^{N} \backslash S$ is a prevalent set. Since $\mathbf{R}^{N} \backslash S \subseteq T^{-1}(0)$, we deduce that $T^{-1}(0)$ is a prevalent set. Since for the statistical structure $\left\{\left(\mathbf{R}^{N}, \mathcal{B}\left(\mathbf{R}^{N}\right), \mu_{\theta}\right): \theta \in \Theta\right\}$ all conditions of the Theorem 6.21 are fulfilled, we claim that there exists an objective consistent estimate of a parameter $\theta$ for the family $\left(\mu_{\theta}\right)_{\theta \in \Theta}$.

Notice that in Theorem 4.1 (see also [19], Theorem 3.1, p. 117) has been considered an example of a strong objective infinite sample consistent estimate of an unknown parameter for a certain statistical structure in the Polish nonlocally compact abelian group $\mathbf{R}^{N}$. In context with this example, we state the following:

Problem 6.1. Let $G$ be a Polish non-locally-compact group admitting an invariant metric. Does there exist a statistical structure $\left\{\left(G, \mathcal{B}(G), \mu_{\theta}\right)\right.$ $: \theta \in \Theta\}$ with $\operatorname{card}(\Theta)=2^{\aleph_{0}}$ for which there exists a strong objective consistent estimate of a parameter $\theta$ ?

\section{On Objective and Strong Objective Consistent Estimates of an Unknown Parameter in a Compact Polish Group $\{0,1\}^{N}$}

Let $x_{1}, x_{2}, \cdots, x_{k}, \cdots$ be an infinite sample obtained by coin tosses. Then the statistical structure described this experiment has the form:

$$
\left\{\left(\{0,1\}^{N}, B\left(\{0,1\}^{N}\right), \mu_{\theta}^{N}\right): \theta \in(0,1)\right\},
$$

where $\mu_{\theta}(\{1\})=\theta$ and $\mu_{\theta}(\{0\})=1-\theta$. By virtue of the strong law of large numbers, we have 


$$
\mu_{\theta}^{N}\left(\left\{\left(x_{k}\right)_{k \in N}:\left(x_{k}\right)_{k \in N} \in\{0,1\}^{N} \& \lim _{n \rightarrow \infty} \frac{\sum_{k=1}^{n} x_{k}}{n}=\theta\right\}\right)=1,
$$

for each $\theta \in(0,1)$.

Notice that for each $k \in N, G_{k}=\{0,1\}$ can be considered as a compact group with an addition group operation $(\bmod 2)$. Hence, the space of all infinite samples $G:=\{0,1\}^{N}$ can be presented as an infinite product of compact groups $\left\{G_{k}: k \in N\right\}$, i.e., $G=\prod_{k \in N} G_{k}$. Also, that the group $G$ admits an invariant metric $\rho$, which is defined by $\rho\left(\left(x_{k}\right)_{k \in N},\left(y_{k}\right)_{k \in N}\right)=\sum_{k \in N} \frac{\left|x_{k}-y_{k}(\bmod 2)\right|}{2^{k+1}\left(1+\left|x_{k}-y_{k}(\bmod 2)\right|\right)} \quad$ for $\quad\left(x_{k}\right)_{k \in N}$, $\left(y_{k}\right)_{k \in N} \in G$. It is obvious that the measure $\lambda_{k}$ on $G_{k}$ defined by $\lambda_{k}(\{0\})=\lambda_{k}(\{1\})=1 / 2$ is a probability Haar measure in $G_{k}$ for each $k \in N$ and for the probability Haar measure $\lambda$ in $G$ the following equality $\lambda=\prod_{k \in N} \lambda_{k}$ holds true, equivalently, $\lambda=\mu_{0,5}^{N}$.

By virtue (7.2), we deduce that the set

$$
A(0,5)=\left\{\left(x_{k}\right)_{k \in N}:\left(x_{k}\right)_{k \in N} \in\{0,1\}^{N} \& \lim _{n \rightarrow \infty} \frac{\sum_{k=1}^{n} x_{k}}{n}=0,5\right\},
$$

is prevalence. Since $A(\theta) \subset G \backslash A(0,5)$ for each $\theta \in(0 ; 1) \backslash\{1 / 2\}$, where

$$
A(\theta)=\left\{\left(x_{k}\right)_{k \in N}:\left(x_{k}\right)_{k \in N} \in\{0,1\}^{N} \& \lim _{n \rightarrow \infty} \frac{\sum_{k=1}^{n} x_{k}}{n}=\theta\right\},
$$

we deduce that they all are shy (equivalently, of Haar measure zero) sets. In terms of [7], this phenomena can be expressed in the following form: 
Theorem 7.1. For "almost every" sequence $\left(x_{k}\right)_{k \in N} \in\{0,1\}^{N}$, its Cezaro means $\left(\frac{\sum_{k=1}^{n} x_{k}}{n}\right)_{n \in N}$ converges to 0,5 whenever $n$ tends to $\infty$.

By virtue the strong law of large numbers, we get

Theorem 7.2. Let fix $\theta_{0} \in(0,1)$. For each $\left(x_{k}\right)_{k \in N} \in G$, we set $T\left(\left(x_{k}\right)_{k \in N}\right)=\lim _{n \rightarrow \infty} \frac{\sum_{k=1}^{n} x_{k}}{n}$ if this limit exists and differs from $\theta_{0}$, and $T\left(\left(x_{k}\right)_{k \in N}\right)=\theta_{0}$, otherwise. Then $T$ is a consistent estimate of an unknown parameter $\theta$ for the statistical structure $\left\{\left(G, \mathcal{B}(G), \mu_{\theta}\right): \theta \in \Theta\right\}$.

Remark 7.3. Following Definition 6.9, the estimate $T$ is subjective because $T^{-1}(1 / 2)$ is a prevalent set. Unlike Theorem 6.21 , there does not exist an objective consistent estimate of an unknown parameter $\theta$ for any statistical structure $\left\{\left(G, \mathcal{B}(G), \mu_{\theta}\right): \theta \in \Theta\right\}$ for which $\operatorname{card}(\Theta)>\aleph_{0}$, where $\aleph_{0}$ denotes the cardinality of the set of all natural numbers. Indeed, assume the contrary and let $T_{1}$ be such an estimate. Then, we get the partition $\left\{T_{1}^{-1}(\theta): \theta \in \Theta\right\}$ of the compact group $G$ into Haar ambivalent sets. Since each Haar ambivalent set is of positive $\lambda$-measure, we get that the probability Haar measure $\lambda$ does not satisfy Suslin property provided that the cardinality of an arbitrary family of pairwise disjoint Borel measurable sets of positive $\lambda$-measure in $G$ is not more than countable.

Remark 7.4. Let consider a mapping $F: G \rightarrow[0,1]$ defined by $F\left(\left(x_{k}\right)_{k \in N}\right)=\sum_{k \in N} \frac{x_{k}}{2^{k}}$ for $\left(x_{k}\right)_{k \in N} \in G$. This is a Borel isomorphism between $G$ and $[0,1]$ such that the following equality $\lambda(X)=\ell_{1}(F(X))$ holds true for each $X \in \mathcal{B}(G)$. By virtue the latter relation, for each natural number $m$, the exists a partition $\left\{X_{k}: 1 \leq k \leq m\right\}$ of the group $G$ 
into Haar ambivalent sets such that for each $1 \leq i \leq j \leq m$, there is an isometric Borel measurable bijection $f_{(i, j)}: G \rightarrow G$ such that the set $f_{(i, j)}\left(X_{i}\right) \Delta X_{j}$ is shy, equivalently, of the $\lambda$-measure zero.

By the scheme presented in the proof of the Theorem 6.21, one can get the validity of the following assertions:

Theorem 7.5. Let $\Theta_{1}$ be a subset of the $\Theta$ with $\operatorname{card}(\Theta) \geq 2$. Then, there exists an objective consistent estimate of an unknown parameter $\theta$ for the statistical structure $\left\{\left(G, \mathcal{B}(G), \mu_{\theta}\right): \theta \in \Theta_{1}\right\}$ if and only if $\operatorname{card}\left(\Theta_{1}\right) \leq \aleph_{0}$ and $1 / 2 \notin \Theta_{1}$.

Theorem 7.6. Let $\Theta_{2}$ be a subset of the $\Theta$ with $\operatorname{card}(\Theta) \geq 2$. Then, there exists a strong objective consistent estimate of an unknown parameter $\theta$ for the statistical structure $\left\{\left(G, \mathcal{B}(G), \mu_{\theta}\right): \theta \in \Theta_{2}\right\}$ if and only if $\operatorname{card}\left(\Theta_{2}\right)<\aleph_{0}$ and $1 / 2 \notin \Theta_{2}$.

\section{Acknowledgement}

The first and third authors are partially supported by Shota Rustaveli National Science Fundation's Grant no FR/503/1-30/14.

\section{References}

[1] R. Balka, Z. Buczolich and M. Elekes, Topological Hausdorff dimension and level sets of generic continuous functions on fractals, Chaos Solitons Fractals 45(12) (2012), 1579-1589; MR3000710.

[2] J. R. Christensen, Measure theoretic zero sets in infinite dimensional spaces and applications to differentiability of Lipschitz mappings, Publ. Dep. Math. 10(2) (1973), 29-39; MR0361770.

[3] J. R. Christensen, Topology and Borel Structure, North-Holland Publishing Company, Amsterdam, 1974; MR0348724.

[4] J. Cohen, The earth is round $(p<.05)$, American Psychologist 49(12) (1994), 997-1003.

[5] R. Dougherty, Examples of non-shy sets, Fund. Math. 144 (1994), 73-88; MR1271479.

[6] H. L. Lavoie, S. A. Mulaik and J. H. Steiger, What if there were no Significance Tests?, Lawrence Erlbaum Associates, 1997. 
[7] B. R. Hunt, T. Sauer and J. A. Yorke, Prevalence: A translation-invariant "almost every" on infinite-dimensional spaces, Bulletin (New Series) of the American Mathematical Society 27(2) (1992), 217-238; MR1161274.

[8] Kaisan High Accuracy Calculation, Cauchy distribution (percentile)

$$
\text { http://keisan.casio.com/has10/SpecExec.cgi }
$$

[9] A. B. Kharazishvili, Topologicheskie aspekty teorii mery, (Russian), [Topological Aspects of Measure Theory] Naukova Dumka, Kiev, 1984; MR0784614.

[10] L. Kuipers and H. Niederreiter, Uniform Distribution of Sequences, WileyInterscience [John Wiley \& Sons], New York-London-Sydney, 1974; MR0419394.

[11] J. Mycielski and S. Swierczkowski, On the Lebesgue measurability and the axiom of determinateness, Fund. 54 (1964), 67-71; MR0161788.

[12] J. Mycielski, Some unsolved problems on the prevalence of ergodicity, instability, and algebraic independence, Ulam Quart. 1(3) (1992), 30 ff., approx. 8 pp. (Electronic only); MR1208681.

[13] E. Nadaraya, On non-parametric estimates of density functions and regression curves, Theor. Prob. Appl. 10 (1965), 186-190.

[14] J. Nunnally, The place of statistics in psychology, Educational and Psychological Measurement 20(4) (1960), 641-650.

[15] G. R. Pantsulaia, On separation properties for families of probability measures, Georgian Math. J. 10(2) (2003), 335-342; MR2009981.

[16] G. R. Pantsulaia, Invariant and quasiinvariant measures in infinite-dimensional topological vector spaces, Nova Science Publishers Inc., New York, 2007; MR2527890.

[17] G. Pantsulaia, On a certain partition of the non-locally compact abelian Polish group $R^{N}$, Proc. A. Razmadze Math. Inst. 149 (2009), 75-86; MR2597356.

[18] G. Pantsulaia and M. Kintsurashvili, Why is null hypothesis rejected for "almost every" infinite sample by some hypothesis testing of maximal reliability?, Journal of Statistics: Advances in Theory and Applications 11(1) (2014), 45-70.

$$
\text { http://www.scientificadvances.co.in }
$$

[19] G. Pantsulaia and M. Kintsurashvili, An effective construction of the strong objective infinite sample well-founded estimate, Proc. A. Razmadze Math. Ins. 166 (2014), 113-119.

[20] G. Pantsulaia and M. Kintsurashvili, An objective infinite sample well-founded estimates of a useful signal in the linear one-dimensional stochastic model, Rep. Enlarged Sess. Semin, I. Vekua Appl. Math. 28 (2014), (Accepted).

[21] M. Rosenblatt, Remarks on some nonparametric estimates of a density function, Textit. Ann. Math. Statist. 27 (1956), 832-837; MR0079873. 
[22] E. F. Schuster, Estimation of a probability density function and its derivatives, Ann. Math. Statist. 40 (1969), 1187-1195; MR0247723.

[23] Albert N. Shiryaev, Problems in Probability, Translated by Andrew Lyasoff, Problem Books in Mathematics, Springer, New York, 2012; MR2961901.

[24] S. Solecki, On Haar null sets, Fund. Math. 149(3) (1996), 205-210; MR1383206.

[25] R. M. Solovay, A model of set theory in which every set of reals is Lebesgue measurable, Ann. Math. 92 (1970), 1-56; MR0265151.

[26] I. Sh. Ibramkhallilov and A. V. Skorokhod, On Well-off Estimates of Parameters of Stochastic Processes (in Russian), Naukova Dumka, Kiev, 1980.

[27] Z. Zerakidze, G. Pantsulaia and G. Saatashvili, On the separation problem for a family of Borel and Baire $G$-powers of shift-measures on R, Ukrainian Math. J. 65(4) (2013), 470-485; MR3125005. 